\title{
Boundary contributions to three loop superstring amplitudes
}

\author{
Kowshik Bettadapura ${ }^{1}$, Hai Lin ${ }^{1,2}$ \\ ${ }^{1}$ Yau Mathematical Sciences Center, Tsinghua University, Beijing 100084, China \\ ${ }^{2}$ Department of Mathematical Sciences, Tsinghua University, Beijing 100084, China
}

\begin{abstract}
In type II superstring theory, the vacuum amplitude at a given loop order $g$ can receive contributions from the boundary of the compactified, genus $g$ supermoduli space of curves $\overline{\mathfrak{M}}_{g}$. These contributions capture the long distance or infrared behaviour of the amplitude. The boundary parametrises degenerations of genus $g$ super Riemann surfaces. A holomorphic projection of the supermoduli space onto its reduced space would then provide a way to integrate the holomorphic, superstring measure and thereby give the superstring vacuum amplitude at $g$-loop order. However, such a projection does not generally exist over the bulk of the supermoduli spaces in higher genera. Nevertheless, certain boundary divisors in $\partial \overline{\mathfrak{M}}_{g}$ may holomorphically map onto a bosonic space upon composition with universal morphisms, thereby enabling an integration of the holomorphic, superstring measure here. Making use of ansatz factorisations of the superstring measure near the boundary, our analysis shows that the boundary contributions to the three loop vacuum amplitude will vanish in closed oriented type II superstring theory with unbroken spacetime supersymmetry.
\end{abstract}




\section{Introduction}

A super Riemann surface of genus $g$ is a (1|1)-dimensional supermanifold which is locally a superspace. Its reduced space is a genus $g$, Riemann surface and its module of odd differentials defines a spin structure on the surface. In contrast to punctures on Riemann surfaces however, there can be two types of punctures on a super Riemann surface originating from external states in superstring theory. These are Neveu-Schwarz (NS) type punctures and the Ramond ( $\mathrm{R}$ ) type punctures.

The moduli space of super Riemann surfaces is referred to generally as supermoduli space. It is a subjest of much importance in several areas of mathematics and physics. It provides a unified framework in which to study problems in algebraic geometry, the mathematics of supersymmetry and superconformal quantum field theory. Methods from supergeometry and superanalysis are essential for studying supersymmetric string theory from the viewpoint of supermoduli space. For an early introduction to the mathematics of supergeometry and superanalysis, see [1, 2, 3] and references therein.

In this paper we are interested in superstring amplitudes and the boundary components of supermoduli spaces. More precisely, we aim to understand contributions to the superstring amplitude at $g$ loop order arising from the boundary of the compactified, genus $g$ supermoduli space $\overline{\mathfrak{M}}_{g}$. As an illustration of our ideas we specialise to genus $g=3$ and analyse the boundary contributions to the three loop vacuum amplitude in Type II superstring theory.

More generally, the supermoduli space $\mathfrak{M}_{g, n, n^{\prime}}$ is the moduli space of genus $g$ super Riemann surfaces with $n$ Neveu-Schwarz punctures and $n^{\prime}$ Ramond punctures. In particular, the dimensionality of the supermoduli space is increased by (1|1) for each Neveu-Schwarz puncture and by $\left(1 \mid \frac{1}{2}\right)$ for each Ramond puncture. Two Ramond punctures contribute to one fermionic modulus. These moduli in superstring perturbation theory play the role of the Schwinger parameters in quantum field theory. In the context of a supersymmetric theory, fermionic moduli play the role of supersymmetric partners to the bosonic moduli.

The Mumford isomorphisms in algebraic geometry are a collection of isomorphisms between certain line bundles over the Deligne-Mumford compactification of the moduli space of Riemann surfaces. In bosonic string theory, defined by the path integral with the Polyakov action functional, these isomorphisms can be used to construct the holomorphic string measure [4, 5]. Due to the existence of tachyons, bosonic string theory is not entirely a physical theory, in contrast to its supersymmetric extension - superstring theory. In superstring theory however, the non-compact superstring configuration space is no longer the moduli space of Riemann surfaces but rather its supersymmetric analogue, the supermoduli space. Accordingly, the Mumford isomorphisms can be generalised to isomorphisms between certain line bundles on supermoduli space [6], leading thereby to a construction of the holomorphic, superstring measure [7]. For a construction of this measure in the presence of NS- and R-punctures, see [8] and [9]. 
The superstring measure on the supermoduli space can also be derived from worldsheet superconformal field theory [7, 10, 11, 12, 13, 14, 15]. The integration of this measure over the supermoduli space then gives the superstring amplitude. A chiral splitting procedure is important [10, 16, 14] in the computation of the genus two superstring measure [17]. In the chiral splitting procedure, one introduces loop momenta to write conformal correlators via an integral over loop momenta whose integrand is the product of the left and right chiral conformal blocks. Each chiral block is analytic in the moduli of the surface as well as in the inserted vertex points.

Integration of the genus $g$, bosonic string measure over the Deligne-Mumford compactifcation of the bosonic moduli space, i.e. the moduli space of Riemann surfaces, gives the $g$ loop bosonic string amplitude. Similarly and as mentioned above, integration of the genus $g$, superstring measure gives a contribution to the superstring amplitude at $g$ loop order. The space over which one integrates is the analogue of the compactification of bosonic moduli space, being a compactification of supermoduli space. Such a compactification was sketched by Deligne in a letter to Manin circa 1987, and described recently and in more detail by Witten in [18] and Donagi and Witten in [19]. Expressions for the superstring amplitude in genus $g=0,1$ were obtained by Green and Schwarz [20], and in $g=2$ by D'Hoker and Phong [17]. However, as observed by Witten [21], these expressions ought to receive potentially non-vanishing contributions from the boundary divisors in the compactification of supermoduli space. At two loop order, Witten nevertheless notes that these boundary contributions will vanish. In this paper we note that the boundary contributions of vacuum amplitude at three loop order will also vanish.

From more mathematical perspectives, one of the main results by Donagi and Witten [19, 22 concerns the question of holomorphically projecting the genus $g$ supermoduli space $\mathfrak{M}_{g}$ onto its reduced space. A holomorphic projection $\pi: \mathfrak{M}_{g} \rightarrow \mathcal{S M}_{g}$ would allow for computing the superstring amplitude by firstly integrating along the odd fibers as stipulated by Berezin [1], and then integrating over the spin moduli space $\mathcal{S M}_{g}$. Since the spin moduli space discretely covers the moduli space of Riemann surfaces $\mathcal{M}_{g}$, measures on $\mathcal{S} \mathcal{M}_{g}$ can be reduced to measures on $\mathcal{M}_{g}$ by summing over the spin structures - a procedure known as GSO projection. This method of reducing the superstring measure to a measure on $\mathcal{M}_{g}$ was used by Green and Schwarz [20] and by D'Hoker and Phong [17] in their derivation of the superstring amplitude to loop orders zero, one and two. Donagi and Witten found however that $\mathfrak{M}_{g}$ cannot be projected onto $\mathcal{S M}_{g}$ for any genus $g \geq 5$, thereby placing a mathematical obstruction to applying known methods to calculate superstring amplitudes to arbitrary loop order. Note, this is in contrast to bosonic string theory where the bosonic string amplitudes are in principle known to any loop order.

Supermoduli space parametrises smooth, super Riemann surfaces. In its compactification, boundary divisors parametrise super Riemann surfaces which can degenerate into two kinds, separating degenerations and non-separating degenerations. These boundary 
divisors can be identified with punctured supermoduli spaces of generally lower genera through a process known as clutching, as studied in a more classical setting in [23]. The boundary contributions to superstring amplitudes involve integrating the superstring measure over these boundary divisors. In this paper we look at these boundary contributions along boundary divisors in the genus $g=3$ supermoduli space. As observed earlier, there do not exist holomorphic projections of supermoduli in genus $g \geq 5$. In genus $g=3$ there does exist a projection, but it is not holomorphic - it is singular along the hyperelliptic locus [21]. As such, although one can use super period matrix [21, 24] as a basis, it is unclear as to how to apply D'Hoker and Phong's integration procedure over the genus $g=3$ supermoduli space. As mentioned above, we have largely considered boundary components of the supermoduli space, which involve supermoduli spaces of lower genera.

The organisation of this paper is as follows. In Section 2, we describe the boundary components of supermoduli space. This section is divided into three subsections which emphasise perspectives from algebraic geometry, from gluing in local, geometric models, and from superstring worldsheet theory. Section 3 is devoted to superstring measures and contributions to the amplitude from the boundary of supermoduli space. This section is divided into five subsections offering perspectives on superstring measures and amplitudes from supergeometry and sheaf theory in algebraic geometry. We analyse the genus three case as a detailed example. In Section 4 we discuss our results and draw conclusions. In the interests of being self-contained, we give a brief overview of the compactification of the moduli space of Riemann surfaces with and without spin structures in Appendix A.

\section{Boundary of supermoduli spaces}

In this section we analyse the boundary of supermoduli space and related aspects. The boundary parametrises super Riemann surfaces with prescribed degenerations. That is, super Riemann surfaces which develop nodes of Neveu-Schwarz (NS) or Ramond (R) type. As described in [18], there are two distinct types of degenerations of a super Riemann surface, referred to as separating and non-separating degenerations. The construction of the boundary of supermoduli space parallels that of the boundary of the Deligne-Mumford compactification of the moduli space of Riemann surfaces in [23] and [25]. In Section 2.1 we analyse the boundary components of supermoduli space parametrising degenerating super Riemann surfaces. We emphasise the role of clutching morphisms, among other things. Section 2.2 reviews gluing formulae for these degenerating surfaces at Neveu-Schwarz and Ramond nodes. In Section 2.3 we describe the relation between the superstring amplitudes and the boundary of the supermoduli spaces. 


\subsection{Boundary components via clutching}

Like the objects it parametrises, the moduli space of genus $g$ super Riemann surfaces $\mathfrak{M}_{g}$ is a superspace. Its reduced space $\mathcal{S} \mathcal{M}_{g}$ parametrises Riemann surfaces with spin structure. As such, its compactification might resemble that of the spin moduli space described by Cornalba [26] and which in turn resembles the Deligne-Mumford compactification [25]. And indeed, the compactification of $\mathfrak{M}_{g}$ is similar to that of $\mathcal{M}_{g}$. To be self-contained, we give a brief overview of the compactifications of the ordinary bosonic moduli space $\mathcal{M}_{g}$ and spin moduli space $\mathcal{S} \mathcal{M}_{g}$ in Appendix A.

Denote by $\mathfrak{M}_{g, n, n^{\prime}}$ the moduli space of super Riemann surfaces of genus $g$ with $n$-many NS-punctures and $n^{\prime}$-many R-punctures. We use a prime in denoting the $\mathrm{R}$ puncture number so as to distinguish them from the NS puncture number. The reduced space of $\mathfrak{M}_{g, n, n^{\prime}}$ is $\mathcal{S} \mathcal{M}_{g, n+n^{\prime}}$. If $n=n^{\prime}=0$, we will just write $\mathfrak{M}_{g}$ rather than $\mathfrak{M}_{g, 0,0^{\prime}}$ for brevity. Similarly, if either $n=0$ or $n^{\prime}=0$, the respective subscript will be omitted. As with the case of spin moduli space, the parity of spin structures induces a decomposition of the supermoduli space: $\mathfrak{M}_{g, n, n^{\prime}}=\mathfrak{M}_{g, n, n^{\prime} ;+} \cup \mathfrak{M}_{g, n, n^{\prime} ;-}$, where + or - denotes even or odd for the parity of the spin structure. It is $\mathfrak{M}_{g, n, n^{\prime} ;+}$ which is of primary interest in superstring theory. But as illustrated in the boundary components of spin moduli space in Appendix A, the boundary of $\partial \overline{\mathfrak{M}}_{g, n, n^{\prime} ;+}$ will inevitably involve odd spin structures. Now in contrast to Neveu-Schwarz punctures, there must always be an even number of Ramond punctures, i.e., $n^{\prime}$ is an even integer. In genus $g \geq 2$, appropriately applying the Riemann-Roch theorem reveals the dimension:

$$
\operatorname{dim} \mathfrak{M}_{g, n, n^{\prime}}=3 g-3+n+n^{\prime} \mid 2 g-2+n+\frac{1}{2} n^{\prime} .
$$

In analogy with stable curves, compactifying $\mathfrak{M}_{g, n, n^{\prime}}$ involves allowing super Riemann surfaces to develop nodes of Neveu-Schwarz or Ramond type. The boundary components

$\partial \overline{\mathfrak{M}}_{g, n, n^{\prime}} \subset \overline{\mathfrak{M}}_{g, n, n^{\prime}}$ ought then be similar to those of $\overline{\mathcal{M}}_{g, n}$ from (A.1). This is indeed the case along the Neveu-Schwarz nodes. As such, components of $\partial \overline{\mathfrak{M}}_{g, n, n^{\prime}}$ are given by the analogue of $\alpha$ - and $\beta$-type clutchings in (A.1), (A.2) and (A.3) for super Riemann surfaces along NS nodes. This leads now to the following clutching morphisms for the boundary component of the even part of supermoduli space:

$$
\overline{\mathfrak{M}}_{g_{1}, n_{1}+1, n_{1}^{\prime} ; \pm} \times \overline{\mathfrak{M}}_{g_{2}, n_{2}+1, n_{2}^{\prime} ; \pm} \stackrel{\alpha( \pm, \pm)}{\longrightarrow} \overline{\mathfrak{M}}_{g, n, n^{\prime} ;+} \quad \text { and } \quad \overline{\mathfrak{M}}_{g-1, n+2, n^{\prime} ;+} \stackrel{\beta^{+}}{\longrightarrow} \overline{\mathfrak{M}}_{g, n, n^{\prime} ;+}
$$

where $g_{1}+g_{2}=g, n_{1}+n_{2}=n$ and $n_{1}^{\prime}+n_{2}^{\prime}=n^{\prime}$. There are similar clutching morphisms to (2.2) describing the boundary of the odd part of supermoduli space. Note that accordingly we have the inclusions,

$$
\operatorname{im} \alpha \subset \partial \overline{\mathfrak{M}}_{g, n, n^{\prime}} \subset \overline{\mathfrak{M}}_{g, n, n^{\prime}}, \quad \operatorname{im} \beta \subset \partial \overline{\mathfrak{M}}_{g, n, n^{\prime}} \subset \overline{\mathfrak{M}}_{g, n, n^{\prime}}
$$

where $\alpha$ and $\beta$ are as in (2.2). We sometimes denote $\alpha=\alpha_{g_{1}, g_{2}}$ and $\beta=\beta_{g-1}$ with the subscript indicating the genus; and sometimes we omit the subscript for brevity. 
If $\mathcal{D}_{N S ; \text { sep. and }} \mathcal{D}_{N S ; n s e p}$. in $\partial \overline{\mathfrak{M}}_{g, n, n^{\prime}}$ denote the divisors parametrising separating and non-separating degenerations along NS-nodes, then:

$$
\mathcal{D}_{N S ; s e p . ; \pm} \cong \overline{\mathfrak{M}}_{g_{1}, n_{1}+1, n_{1}^{\prime} ; \pm} \times \overline{\mathfrak{M}}_{g_{2}, n_{2}+1, n_{2}^{\prime} ; \pm} \quad \text { and } \quad \mathcal{D}_{N S ; n s e p . ;+} \cong \overline{\mathfrak{M}}_{g-1, n+2, n^{\prime} ;+} .
$$

Note $\mathcal{D}_{N S ; \text { sep. }}=\mathcal{D}_{N S ; \text { sep.;+ }} \cup \mathcal{D}_{N S ; s e p . ;-}$ and similarly for $\mathcal{D}_{N S ; n s e p .}$. These divisors form the boundary components of $\overline{\mathfrak{M}}_{g, n . n^{\prime}}$ corresponding to degenerations along NS nodes. The divisors parametrising degenerations along Ramond nodes cannot be so elegantly described however. As discussed by Witten [18], the divisors $\mathcal{D}_{R ; \text { sep. }}$ and $\mathcal{D}_{R ; n \text { sep }}$ in $\partial \overline{\mathfrak{M}}_{g, n, n^{\prime}}$ parametrising separating and non-separating degenerations along Ramond nodes are fibered over the expected boundary components with fermionic fiber. More generally, if $\mathfrak{X}$ is a supermanifold with boundary $\partial \mathfrak{X}$, the boundary $\partial \mathfrak{X} \subset \mathfrak{X}$ will have codimension$(1 \mid 0)$. Dimension counting now reveals, along a separating Ramond degeneration:

$$
\begin{aligned}
\operatorname{dim} \mathfrak{M}_{g, n, n^{\prime}}-\left(\operatorname{dim} \mathfrak{M}_{g_{1}, n_{1}, n_{1}^{\prime}}\right. & \left.+\operatorname{dim} \mathfrak{M}_{g_{2}, n_{2}, n_{2}^{\prime}}\right) \\
& =n^{\prime}-\left(n_{1}^{\prime}+n_{2}^{\prime}\right)+3 \mid \frac{1}{2}\left(n^{\prime}-\left(n_{1}^{\prime}+n_{2}^{\prime}\right)\right)+2
\end{aligned}
$$

where we have taken $g=g_{1}+g_{2}$ and $n=n_{1}+n_{2}$. Since the number of Ramond punctures must be even, and since $n^{\prime}<n_{1}^{\prime}+n_{2}^{\prime}$, the dimension formula in (2.5) only makes sense when $n^{\prime}+2=n_{1}^{\prime}+n_{2}^{\prime}$. Evidently, the divisor $\mathcal{D}_{R ; s e p .} \subset \partial \overline{\mathfrak{M}}_{g, n, n^{\prime}}$ parametrising separating degeneration along Ramond punctures is a (0|1)-dimensional fibration over $\overline{\mathfrak{M}}_{g_{1}, n_{1}, n_{1}^{\prime}} \times \overline{\mathfrak{M}}_{g_{2}, n_{2}, n_{2}^{\prime}}$. A similar analysis in the non-separating case $\mathcal{D}_{R ; n s e p .} \subset \partial \overline{\mathfrak{M}}_{g, n, n^{\prime}}$ reveals it will fiber over $\overline{\mathfrak{M}}_{g-1, n, n^{\prime}+2}$ with (0|1)-dimensional fibers. And so, the boundary components $\mathcal{D}_{R ; s e p .}, \mathcal{D}_{R ; n s e p .} \subset \partial \overline{\mathfrak{M}}_{g, n . n^{\prime}}$ parametrising separating and non-separating Ramond degenerations can be realised as fibrations:

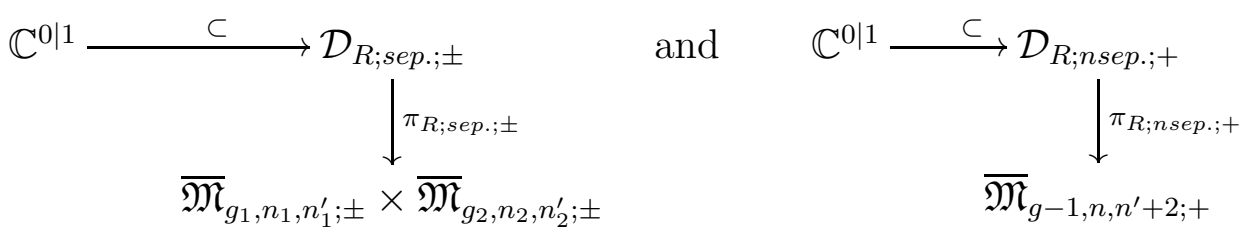

where $g_{1}+g_{2}=g, n_{1}+n_{2}=n$ and $n_{1}^{\prime}+n_{2}^{\prime}=n^{\prime}+2$. Note the contrast with NS-case in (2.4). As we will see in Section 2.2, this contrast will be apparent in the gluing laws along the nodes in the following sense: there are no free, odd parameters in the gluing laws along NS nodes. As in the Neveu-Schwarz case, the divisors parametising Ramond degenerations decompose according to the parity of spin structures, i.e., $\mathcal{D}_{R ; \text { sep. }}=\mathcal{D}_{R ; \text { sep.;+ }} \cup \mathcal{D}_{R ; \text { sep.;- }}$, and similarly for $\mathcal{D}_{R ; \text { sep. }}$

We can now write the boundary of supermoduli space as follows:

$$
\partial \overline{\mathfrak{M}}_{g, n, n^{\prime}}=\partial \overline{\mathfrak{M}}_{g, n, n^{\prime} ; N S} \cup \partial \overline{\mathfrak{M}}_{g, n, n^{\prime} ; R}
$$

The compactified supermoduli space is now the union of the bulk with the boundary,

$$
\overline{\mathfrak{M}}_{g, n, n^{\prime}}=\mathfrak{M}_{g, n, n^{\prime}} \cup \partial \overline{\mathfrak{M}}_{g, n, n^{\prime}} .
$$


Another description of the boundary is as a union over irreducible components $\partial \overline{\mathfrak{M}}_{g, n, n^{\prime}}=\bigcup_{j} \Delta_{j}$, where $\Delta_{j}$ denotes an irreducible component. Then to the embedding $\Delta_{j} \subset \overline{\mathfrak{M}}_{g, n, n^{\prime}}$ there is a bundle of normal sections to the embedding over $\Delta_{j}$, denoted $\mathcal{N}_{\Delta_{j}}$. Its sheaf of sections, denoted $\hat{\mathcal{N}}_{\Delta_{j}}$, fits into a short exact sequence

$$
\left.0 \rightarrow T_{\Delta_{j}} \rightarrow T_{\overline{\mathfrak{M}}_{g, n, n^{\prime}}}\right|_{\Delta_{j}} \rightarrow \hat{\mathcal{N}}_{\Delta_{j}} \rightarrow 0
$$

or dually

$$
\left.0 \rightarrow \hat{\mathcal{N}}_{\Delta_{j}}^{*} \rightarrow T_{\mathfrak{M}_{g, n, n^{\prime}}}^{*}\right|_{\Delta_{j}} \rightarrow T_{\Delta_{j}}^{*} \rightarrow 0 .
$$

We denote by $\mathfrak{N}_{\Delta_{j}}$ the fiber of the normal bundle $\mathcal{N}_{\Delta_{j}}$ to the embedding $\Delta_{j} \subset \overline{\mathfrak{M}}_{g, n, n^{\prime}}$. This gives a fibration of spaces,

$$
\mathfrak{N}_{\Delta_{j}} \rightarrow \mathcal{N}_{\Delta_{j}} \rightarrow \Delta_{j}
$$

As mentioned earlier, the sheaf of sections of $\mathcal{N}_{\Delta_{j}}$ over $\Delta_{j}$ is $\hat{\mathcal{N}}_{\Delta_{j}}$.

As in the case of Riemann surfaces with marked points, we can form a forgetful map on super Riemann surfaces,

$$
\overline{\mathfrak{M}}_{g, n+1, n^{\prime}} \stackrel{p}{\longrightarrow} \overline{\mathfrak{M}}_{g, n, n^{\prime}},
$$

given by forgetting the $(n+1)$-th NS puncture and stabilizing the resulting punctured super Riemann surface. The pull-back along the forgetful map is associated to the insertion of an vertex operator in superconformal field theory. We also denote $p=p_{g}$ with the subscript meaning the genus. Sometimes we omit the subscript for brevity. Composite forgetful maps gives the morphism $\overline{\mathfrak{M}}_{g, n+2, n^{\prime}} \stackrel{p^{2}}{\longrightarrow} \overline{\mathfrak{M}}_{g, n, n^{\prime}}$.

The following are diagrams of morphisms relevant for subsequent constructions in this paper:

and

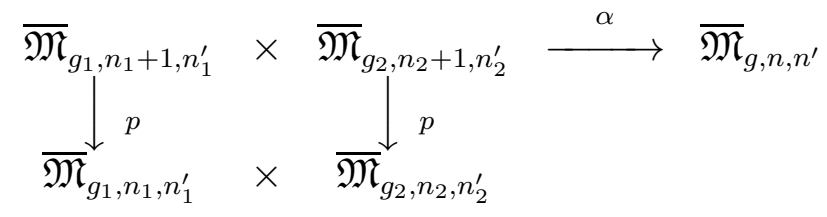

$$
\overline{\mathfrak{M}}_{g-1, n+2, n^{\prime}} \stackrel{\alpha}{\longrightarrow} \overline{\mathfrak{M}}_{g, n, n^{\prime}}
$$

The clutching morphisms are defined on boundary components of supermoduli spaces which parametrise degenerating super Riemann surfaces. These degenerations can be realised in a geometric model involving gluing maps in Section 2.2. They are important for Section 3.3.

In this section we have described the compactification of supermoduli space. In Section 3.2 we consider a 'partial compactification' of supermoduli space. This is in order to ensure that a certain, holomorphic map defined on the bulk will remain holomorphic when extended to the (partial) compactification. 


\subsection{Degenerations and gluing maps in a geometric model}

The degeneration of super Riemann surfaces parametrised by the boundary of supermoduli spaces can be realised in a geometric model via gluing. These models are necessarily local however, in the sense that the gluing map is a local map. It is only defined near the node along which the super Riemann surface degenerates.

As described in [7], we can glue super Riemann surfaces $\mathcal{S}_{\ell}$ and $\mathcal{S}_{r}$ with local coordinates $x \mid \theta$ and $y \mid \psi$, respectively. We glue them so that the gluing happens at the points $a \mid \alpha \in \mathcal{S}_{\ell}$ and $b \mid \beta \in \mathcal{S}_{r}$,

$$
\begin{aligned}
(x-a-\alpha \theta)(y-b-\beta \psi) & =-\varepsilon^{2}=q_{N S}, \\
(y-b-\beta \psi)(\theta-\alpha) & =\varepsilon(\psi-\beta), \\
(x-a-\alpha \theta)(\psi-\beta) & =-\varepsilon(\theta-\alpha), \\
(\theta-\alpha)(\psi-\beta) & =0 .
\end{aligned}
$$

In the language of theoretical physics, the superconformal structures are defined by the vector fields $D_{\theta}=\partial_{\theta}+\theta \partial_{x}$ and $D_{\psi}=\partial_{\psi}+\psi \partial_{y}$ on the surfaces $\mathcal{S}_{\ell}$ and $\mathcal{S}_{r}$ respectively. The gluing formula maps the superconformal coordinates of the left component $\mathcal{S}_{\ell}$ to the superconformal coordinates of the right component $\mathcal{S}_{r}$. The above (2.15) is gluing along the NS punctures and then smoothed out. The $\alpha, \beta$ are fermionic parameters.

The Ramond punctures define divisors on the super Riemann surface [7], of dimension $0 \mid 1$. We can also glue along these divisors in a manner analogous to but more subtly than in (2.15). In the case of a separating Ramond degeneration, the Ramond punctures in $\mathcal{S}_{\ell}$ and $\mathcal{S}_{r}$ are glued and smoothed out. In local coordinates $x \mid \theta$ on $\mathcal{S}_{l}$ and $y \mid \psi$ on $\mathcal{S}_{r}$ the superconformal structures are defined by the vector fields $\partial_{\theta}+x \theta \partial_{x}$ and $\partial_{\psi}+y \psi \partial_{y}$ respectively. The divisors corresponding to the Ramond punctures are defined respectively by $x=0$ and $y=0$; and the gluing is [7]

$$
\begin{aligned}
x y & =q_{R}, \\
\theta & =\zeta \pm \sqrt{-1} \psi .
\end{aligned}
$$

where $q_{R}$ is a bosonic gluing parameter. The free fermionic parameter $\zeta$ parametrizes the fiber of a component of the fibration $\mathcal{D}_{\text {sep.;R }}$, c.f., (2.6). Evidently, each Ramond divisor has fermionic fiber $\mathbb{C}^{0 \mid 1}$.

\subsection{Amplitudes from boundary contributions}

We would like to calculate amplitudes for the scattering of $n+n^{\prime}$ superstring states. These can be calculated by the correlation functions $\left\langle V_{1} \ldots V_{n+n^{\prime}}\right\rangle$ of vertex operators $V_{1}, \ldots, V_{n+n^{\prime}}$ in worldsheet superconformal field theory. We use the RNS formalism with manifest worldsheet supersymmetry to quantise the theory. The superstring worldsheet is manifestly a super Riemann surface and so the amplitude can also be calculated by 
integrating over supermoduli space. To illustrate, consider a form $\hat{F}_{V_{1}, \ldots, V_{n+n^{\prime}}}$ on the supermoduli space

$$
\hat{F}_{V_{1}, \ldots, V_{n+n^{\prime}}}=\int \mathcal{D}(X, B, C, \tilde{B}, \tilde{C}) \exp (-\hat{I}) \prod_{i=1}^{n+n^{\prime}} V_{i}\left(p_{i}\right),
$$

where $X$ denotes worldsheet matter fields which are also the spacetime coordinates, $C, \tilde{C}$ denote worldsheet ghost fields, $B, \tilde{B}$ denote worldsheet antighost fields, $p_{i}$ denote points, and $\hat{I}$ is the action of the worldsheet theory after gauge-fixing. In the simplest formalism we use NS vertex operators of picture number -1 , and Ramond vertex operators of picture number $-1 / 2$.

The genus $g$ total contribution to the scattering amplitude is $\hat{\mathcal{A}}=\left\langle V_{1} \ldots V_{n+n^{\prime}}\right\rangle$. We consider $\hat{\mathcal{A}}$ to be the summation of contributions from the bulk and from the boundary of supermoduli space $\hat{\mathcal{A}}_{\text {bulk }}=\hat{\mathcal{A}}-\mathcal{A}$ and $\hat{\mathcal{A}}_{\text {bndy }}=\mathcal{A}$ respectively. The form restricted to the boundary is

$$
F_{V_{1}, \ldots, V_{n+n^{\prime}}}=\left.\hat{F}_{V_{1}, \ldots, V_{n+n^{\prime}}}\right|_{\partial \overline{\mathfrak{M}}_{g, n, n^{\prime}}} .
$$

The contribution to the amplitude, from the boundary of the supermoduli space, where the super Riemann surfaces degenerate, is given by

$$
\mathcal{A}=\int_{\partial \overline{\mathfrak{M}}_{g, n, n^{\prime}}} \int_{\mathfrak{N}} F_{V_{1}, \ldots, V_{n+n^{\prime}}}
$$

Here $\mathfrak{N}$ is the fiber of the normal bundle to the boundary divisor, discussed in (2.11).

\section{Boundary contributions and amplitudes}

In this section we analyse contributions to amplitudes from the boundary of supermoduli spaces. We begin in Section 3.1 by presenting an overview of the Berezinian of bundles over supermoduli space. We present Lemma 3.1 concerning a particular class of Berezinians. These are relevant in the formulation of the super Mumford isomorphisms and forms which define the superstring measures. In Section 3.2 we investigate contributions from the boundary of supermoduli space for a general genus. This involves firstly constructing a holomorphic map from a partial compactification of supermoduli space in genus two; and secondly, using this map as a building block to extract the aforementioned boundary contributions. In Section 3.3 we use the geometric model of gluing maps to illustrate the pole behaviour of the super Mumford form near the boundary divisors. In Section 3.4 we overview the notion of integration on supermanifolds more generally, with the aim to apply these notions to compute superstring amplitudes through integration over supermoduli spaces. We present a useful integration formula (3.53) which

will be used. Section 3.5 serves as an example of these formalisms for the three loop vacuum amplitude. 


\subsection{Super Mumford isomorphisms and forms}

A supermanifold $\mathfrak{X}$ is a space which is modelled on the data of a manifold $X$ and a vector bundle $T_{X,-}^{*}$ on $X$, thought of as the module of 'odd' differentials or 'fermionic parameters'. The subscript + or - denote even or odd respectively, in this section. We can also think of $T_{X,-}^{*}$ as a locally free sheaf on $X$. The space $X$ is itself referred to as the reduced space of $\mathfrak{X}$. The prototype supermanifold associated to $\left(X, T_{X,-}^{*}\right)$ is the split model, which is the locally ringed space $\left(X, \wedge^{\bullet} T_{X,-}^{*}\right)$. Its dimension is defined by a pair $(p \mid q)$ where $p=\operatorname{dim} X$ and $q=\operatorname{rank} T_{X,-}^{*}$. More generally, a supermanifold modelled on $\left(X, T_{X,-}^{*}\right)$ is a locally ringed space $\mathfrak{X}=\left(X, \mathcal{O}_{\mathfrak{X}}\right)$ where $\mathcal{O}_{\mathfrak{X}}$ is a sheaf of (local) supercommutative algebras on $X$, referred to as the structure sheaf. It is, additionally, required to be locally isomorphic to $\wedge^{\bullet} T_{X,-}^{*}$ as sheaves over $X$. The dimension of $\mathfrak{X}$ coincides with the dimension of the split model. If $\mathfrak{X}$ is isomorphic to the split model, it is split; otherwise, it is non-split. A more relevant and weaker condition for the purposes of physics is a (holomorphic) projection or fibration of $\mathfrak{X}$ over its reduced space $X$. As we will discuss in sections to follow, the existence of a projection allows for the reduction of measures on superspace to measures on the reduced space where classical methods of integration can be applied. Note, if $\mathfrak{X}$ is split, then it is projected; but not necessarily conversely.

Since the structure sheaf $\mathcal{O}_{\mathfrak{X}}$ of a supermanifold $\mathfrak{X}=\left(X, \mathcal{O}_{\mathfrak{X}}\right)$ is a sheaf of supercommutative algebras, it is globally $\mathbb{Z}_{2}$-graded. Hence we can write $\mathcal{O}_{\mathfrak{X}}=\mathcal{O}_{\mathfrak{X},+} \oplus \mathcal{O}_{\mathfrak{X},-}$ as $\mathcal{O}_{\mathfrak{X},+}$-modules. The tangent sheaf can be graded compatibly and so we have

$$
T_{\mathfrak{X}}=T_{\mathfrak{X},+} \oplus T_{\mathfrak{X},-} \cdot
$$

If $\mathcal{J}=\mathcal{O}_{\mathfrak{X},-} \cdot \mathcal{O}_{\mathfrak{X}} \subset \mathcal{O}_{\mathfrak{X}}$ denotes the fermionic ideal, then the structure sheaf of the reduced space $X$ is $\mathcal{O}_{X}=\mathcal{O}_{\mathfrak{X}} / \mathcal{J}$. If $\mathfrak{X}$ is modelled on $\left(X, T_{X,-}^{*}\right)$ then $T_{\mathfrak{X}, \pm} \cong T_{X, \pm} \bmod \mathcal{J}$, where $T_{X,+}=T_{X}$ is the tangent sheaf of $X$ and $T_{X,-}$ are the 'odd' tangent vectors. Hence, if $\mathfrak{X}$ is a supermanifold modelled on $\left(X, T_{X,-}^{*}\right)$ with tangent sheaf as in (3.1), its Berezinian will then be given by

$$
\operatorname{Ber} \mathfrak{X}=\operatorname{Ber} T_{\mathfrak{X}}^{*} \cong \frac{\omega_{X}}{\operatorname{det} T_{X,-}^{*}}=\omega_{X} \otimes \operatorname{det} T_{X,-} \quad \bmod \mathcal{J}
$$

where $\omega_{X}=\operatorname{det} T_{X,+}^{*}$ is the canonical bundle of $X$. Here, forming the denominator means tensoring by its dual.

A super Riemann surface of genus $g$ is a (1|1)-dimensional supermanifold $\mathcal{S}$ modelled on a Riemann surface $C$ and a spin structure $T_{C,-}^{*}$ as its module of odd differentials. Spin structures are also referred to as theta characteristics [26] in the language of algebraic geometry. Recall that a line bundle on a Riemann surface is an ordinary spin structure if its quadratic tensor power is isomorphic to the canonical bundle. That the module of odd differentials must be a spin structure follows from a more general characterisation: a super Riemann surface $\mathcal{S}$ is a supermanifold with $C$ as its reduced space and a choice 
of nowhere integrable distribution $\mathcal{D} \subset T_{\mathcal{S}}$, where $\mathcal{D}$ is generated by the superconformal vector fields on the super Riemann surface. In denoting the superconformal vector field by $D_{\theta}=\partial_{\theta}+\theta w(x) \partial_{x}$, see that $D_{\theta}^{2}=w(x) \partial_{x}$. In particular, the function $w(x)$ has zeros along the Ramond punctures where $D_{\theta}^{2}=0$. We denote the divisors for Neveu-Schwarz or Ramond punctures on the super Riemann surface as $\mathcal{P}_{i}$ or $\mathcal{F}_{i}$ respectively in our convention, and their sum by $\mathcal{P}=\sum_{i=1}^{n} \mathcal{P}_{i}, \mathcal{F}=\sum_{i=1}^{n^{\prime}} \mathcal{F}_{i}$. On a super Riemann surface then with $n$ Neveu-Schwarz punctures and $n^{\prime}$ Ramond punctures, the distribution $\mathcal{D}$ sits in a short exact sequence

$$
0 \rightarrow \mathcal{D} \rightarrow T_{\mathcal{S}} \rightarrow \mathcal{D}^{\otimes 2} \otimes \mathcal{O}(\mathcal{P}+\mathcal{F}) \rightarrow 0
$$

One might alternatively and equivalently denote super Riemann surfaces by the pair $(\mathcal{S}, \mathcal{D})$ and define a generalised spin structure by $\left.\mathcal{D}^{s} \stackrel{\text { def }}{=} \mathcal{D}^{*}\right|_{C}$, following [18]. Here $\mathcal{D}$ is a choice of distribution on $\mathcal{S}$, and $\mathcal{D}^{s}$ is a generalised spin structure on the reduced space $C$. If $\mathcal{S}$ is a (1|1)-dimensional supermanifold over a Riemann surface $C$, then every distribution $\mathcal{D} \subset T_{\mathcal{S}}$ satisfying (3.3) is in bijective correspondence with generalised spin structures $\mathcal{D}^{s}$ on $C$.

Consequently, viewing $\mathfrak{M}_{g, n, n^{\prime}}$ as a supermanifold and using the description of tangent spaces of supermanifolds (modulo the fermionic ideal), at any isomorphism class $[(\mathcal{S}, \mathcal{D})] \in \mathfrak{M}_{g, n, n^{\prime}}$ we have:

$$
\begin{aligned}
\left.T_{\mathfrak{M}_{g, n, n^{\prime}},+}\right|_{[(\mathcal{S}, \mathcal{D})]} & =H^{1}\left(C, T_{C} \otimes \mathcal{O}(-\mathcal{P}-\mathcal{F})\right), \\
\left.T_{\mathfrak{M}_{g, n, n^{\prime}},-}\right|_{[(\mathcal{S}, \mathcal{D})]} & =H^{1}\left(C, \mathcal{D}^{s *}\right)
\end{aligned}
$$

where $\mathcal{D}^{s}$ is the spin structure on $C$ which is uniquely associated to the distribution $\mathcal{D}$ and $\mathcal{D}^{s *}$ is its dual.

In (3.2) we have a general expression for the Berezinian of a supermanifold. When $\mathfrak{X}=\mathfrak{M}_{g, n, n^{\prime}}$ is a supermoduli space, its tangent bundle is described in (3.4). Then by Serre duality we find, at a super Riemann surface class $[(\mathcal{S}, \mathcal{D})]$ with underlying Riemann surface $C$, its cotangent space is given by:

$$
\left.T_{\mathfrak{M}_{g, n, n^{\prime}},+}^{*}\right|_{[(\mathcal{S}, \mathcal{D})]}=H^{0}\left(C, \omega_{C}^{\otimes 2} \otimes \mathcal{O}(\mathcal{P}+\mathcal{F})\right),\left.\quad T_{\mathfrak{M}_{g, n, n^{\prime}},-}^{*}\right|_{[(\mathcal{S}, \mathcal{D})]}=H^{0}\left(C, \omega_{C} \otimes \mathcal{D}^{s}\right)
$$

where $\omega_{C}$ is the canonical bundle on the curve $C$. Using (3.2) now, the fiber of the Berezinian of $\mathfrak{M}_{g, n, n^{\prime}}$ at a point $[(\mathcal{S}, \mathcal{D})]$ on supermoduli space is:

$$
\text { Ber }\left.\mathfrak{M}_{g, n, n^{\prime}}\right|_{[(\mathcal{S}, \mathcal{D})]} \cong \frac{H^{0}\left(C, \omega_{C}^{\otimes 2} \otimes \mathcal{O}(\mathcal{P}+\mathcal{F})\right)}{H^{0}\left(C, \omega_{C} \otimes \mathcal{D}^{s}\right)}
$$

modulo the fermionic ideal in $\mathcal{O}_{\mathfrak{M}_{g, n, n^{\prime}}}$.

The classical Mumford isomorphism between certain line bundles over the moduli space of Riemann surfaces can be generalised to isomorphisms between certain 
Berezinians over the supermoduli space $\mathfrak{M}_{g}$. With $L_{g}$ a line bundle with fiber $\left.L_{g}\right|_{[C]}=$ $\operatorname{det} H^{0}\left(C, \omega_{C}\right)$, the bosonic Mumford isomorphism is $L_{g}{ }^{n} \cong L_{g}^{\otimes\left(6 n^{2}-6 n+1\right)}$. Over super Riemann surfaces we can define a line bundle or, more generally, a sheaf $L_{g}^{3 / 2}$ with fiber $\left.L_{g}^{3 / 2}\right|_{[(\mathcal{S}, \mathcal{D})}=\operatorname{det} H^{0}\left(C, \omega_{C} \otimes \mathcal{D}^{s}\right)$. In supergeometry, the Berezinian plays the role of the determinants and so taking Berezinians and tensor powers leads to the following.

Lemma 3.1. Over a genus $g$ super Riemann surface class $[(\mathcal{S}, \mathcal{D})]$ with underlying Riemann surface $C$, we can define a family of sheaves $L_{g}{ }^{n, m}$ with fiber

$$
\left.L_{g}{ }^{n, m}\right|_{[(\mathcal{S}, \mathcal{D})]}=\operatorname{Ber}\left\{H^{0}\left(C, \omega_{C}^{\otimes n}\right) \oplus H^{0}\left(C,\left(\omega_{C} \otimes \mathcal{D}^{s}\right)^{\otimes m}\right)\right\} .
$$

Then

$$
L_{g}^{n, m} \cong L_{g}^{n} \otimes\left(L_{g}^{\frac{3 m}{2}}\right)^{*}
$$

Proof. Since $\left(\mathcal{D}^{s}\right)^{\otimes 2} \cong \omega_{C}$, we can set $\mathcal{D}^{s}=\omega_{C}^{1 / 2}$ and thereby identify $H^{0}\left(C,\left(\omega_{C} \otimes\right.\right.$ $\left.\mathcal{D}^{s}\right)^{\otimes m}$ ) with $L_{g}^{\frac{3 m}{2}}$. Hence we have that $L_{g}{ }^{n, m} \cong L_{g}{ }^{n} \otimes\left(L_{g}^{3 m / 2}\right)^{*}$.

We see that (3.6) is a particular case of (3.7) for $(n, m)=(2,1)$, and hence Ber $\mathfrak{M}_{g}=$ $L_{g}{ }^{2,1}$. The Mumford isomorphism realises the line bundles $L_{g}{ }^{n}$ as a certain tensor powers of $L_{g}=L_{g}{ }^{1}$. With $3 m / 2=m^{\prime}+1 / 2$ in (3.8), for $m^{\prime}$ the integral part, we obtain from the classical Mumford isomorphism,

$$
\begin{aligned}
L_{g}{ }^{n, m} & \cong L_{g}{ }^{n} \otimes\left(L_{g}^{m^{\prime}+\frac{1}{2}}\right)^{*} \\
& \cong L_{g}^{\otimes\left(6 n^{2}-6 n+1\right)} \otimes\left(L_{g}^{\otimes\left(6 m^{\prime 2}-1 / 2\right)}\right)^{*} .
\end{aligned}
$$

For $(n, m)=(2,1)$, from (3.8) and (3.9) above we find,

$$
\text { Ber } \mathfrak{M}_{g} \cong L_{g}^{\otimes 13} \otimes\left(L_{g}^{\otimes \frac{11}{2}}\right)^{*} \cong\left(L_{g}^{3 / 2}\right)^{5} .
$$

This is the Mumford isomorphism for the Berezinian of supermoduli space, i.e., the super Mumford isomorphism. Now in (3.10) we see that the right-hand side is a tensor product of powers of the line bundle $L_{g}=L_{g}{ }^{1}$ and $\left(L_{g}\right)^{*}=L_{g}{ }^{-1}$.

There is a variant of this description. In [8] and [6], the authors use the Mumford isomorphism over supermoduli space in a slightly different form to that in (3.10). Note from (3.4) that

$$
\left(\text { Ber } T_{\mathfrak{M}_{g}}\right)^{*} \cong L_{g}^{2} \otimes\left(L_{g}^{\frac{1}{2}}\right)^{*} \cong L_{g}^{3 / 2}
$$

Hence we find

$$
\text { Ber } \mathfrak{M}_{g} \cong\left(L_{g}^{3 / 2}\right)^{5} \cong\left(\left(\operatorname{Ber} T_{\mathfrak{M}_{g}}\right)^{*}\right)^{5}=\left(\operatorname{Ber} T_{\mathfrak{M}_{g}}\right)^{-5}
$$


The isomorphism Ber $\mathfrak{M}_{g} \cong\left(\operatorname{Ber} T_{\mathfrak{M}_{g}}\right)^{-5}$ in (3.12) is used by Witten [8] for superstring perturbation theory. We have seen that this also follows from our Lemma 3.1.

To understand the relation to the super Mumford form, firstly observe that the super Mumford isomorphism in (3.12) is equivalent to requiring Ber $T_{\mathfrak{M}_{g}}^{*} \otimes\left(\text { Ber } T_{\overline{\mathfrak{M}}_{g}}\right)^{5}$ be holomorphically trivial, meaning Ber $T_{\mathfrak{M}_{g}}^{*} \otimes\left(\text { Ber } T_{\overline{\mathfrak{M}}_{g}}\right)^{5} \cong \mathcal{O}_{\overline{\mathfrak{M}}_{g}}$. The genus $g$ super Mumford form, denoted $\Psi_{g}$, is then a global section of this trivial bundle, i.e., that $\Psi_{g} \in \Gamma\left(\overline{\mathfrak{M}}_{g}\right.$, Ber $\left.T_{\overline{\mathfrak{M}}_{g}}^{*} \otimes\left(\operatorname{Ber} T_{\overline{\mathfrak{M}}_{g}}\right)^{5}\right)$. These isomorphisms and forms can be generalised to the case where the super Riemann surfaces have $n$ Neveu-Schwarz and $n^{\prime}$ Ramond punctures, leading to super Mumford forms $\Psi_{g, n, n^{\prime}}$ over $\overline{\mathfrak{M}}_{g, n, n^{\prime}}$.

\subsection{Boundary contributions and partial compactifications of supermoduli spaces}

The vacuum superstring amplitude at $g$ loop order is an integral of the superstring measure, which ought to be computed not over $\mathfrak{M}_{g}$ but its compactification $\overline{\mathfrak{M}}_{g}$. The total, $g$ loop superstring amplitude therefore receives contributions from the bulk $\mathfrak{M}_{g}$ and from the boundary $\partial \overline{\mathfrak{M}}_{g}$. Witten [21] looks at the boundary in genus $g=2$ and observes that contributions to D'Hoker and Phong's derivation of the superstring amplitude to two-loop order will be vanishing. This is consistent with the vanishing of the two-loop vacuum amplitude obtained by D'Hoker and Phong. In this paper we look at boundary contributions to the three-loop and higher loop amplitudes. As discussed in previous sections, the boundary of supermoduli space has codimension (1|0) and parametrises super Riemann surfaces of lower genus. In particular while $\overline{\mathfrak{M}}_{g, n, n^{\prime}}$, for a given $\left(g, n, n^{\prime}\right)$ may have complications related to projectability of its bulk $\mathfrak{M}_{g, n, n^{\prime}}$, some of its lower genus boundary components may nevertheless fiber over their reduced space with odd dimensional fibers or holomorphically map to a bosonic space. Now for dimensional reasons, $\mathfrak{M}_{g}$ is split for genus $g=0,1$, and hence projected, i.e., can be holomorphically fibered over its reduced space. In contrast, Donagi and Witten [19] showed that: $\mathfrak{M}_{g, n, n^{\prime}}$ (and so also its compactification $\overline{\mathfrak{M}}_{g, n, n^{\prime}}$ ) will be non-projected for

$$
g-1 \geq n+n^{\prime} \geq 1 \text {. }
$$

Note in particular that $\mathfrak{M}_{2,1}$ will be non-projected. Surprisingly however, $\mathfrak{M}_{2 ;+}$ in fact is projected as ilustrated by D'Hoker and Phong in their computation of the superstring vacuum amplitude at two loop order.

The projection $\mathfrak{M}_{2 ;+} \rightarrow \mathcal{S M}_{2 ;+} \rightarrow \mathcal{M}_{2}$ constructed by D'Hoker and Phong uses the identification of $g=2$ super Riemann surfaces $\mathcal{S}$ with their period matrices $\Omega(\mathcal{S})$; and a formula relating the period matrix $\Omega(\mathcal{S})$ to the period matrix of the underlying Riemann surface $C$, denoted $\Omega(C)$. In a particular gauge, termed split gauge, this formula identifies $\Omega(\mathcal{S})$ with $\Omega(C)$, thereby leading to the holomorphic projection $\mathcal{S} \mapsto$ $\Omega(\mathcal{S}) \equiv \Omega(C) \mapsto C$. Since $\mathfrak{M}_{2,1}$ and, by extension, $\overline{\mathfrak{M}}_{2,1}$ are non-projected by (3.13), 
an analogous procedure to that performed by D'Hoker and Phong will not result in a holomorphic projection. It results instead in a meromorphic projection $\mathfrak{M}_{2,1 ;+} \rightarrow$ $\mathcal{S M}_{2,1 ;+}$. It is singular along the divisor parametrising NS degenerations. However, in composing the projection $\pi_{2 ;+}: \mathfrak{M}_{2 ;+} \rightarrow \mathcal{S} \mathcal{M}_{2 ;+}$ with the forgetful map $\mathfrak{M}_{2,1 ;+} \stackrel{p}{\rightarrow} \mathfrak{M}_{2 ;+}$, we obtain a holomorphic map

$$
q_{2,1 ;+}: \mathfrak{M}_{2,1 ;+} \stackrel{p}{\longrightarrow} \mathfrak{M}_{2 ;+} \stackrel{\pi_{2}}{\longrightarrow} \mathcal{S M}_{2 ;+}
$$

In this way, measures on $\mathfrak{M}_{2,1 ;+}$ can be reduced to measures on $\mathcal{S M}_{2 ;+}$ and, upon summing over the even spin structures (GSO projection), to measures on $\mathcal{M}_{2}$. Now more generally, if $\mu$ is an integration measure on $\mathfrak{M}_{2,1 ;+}$, then along $q_{2,1 ;+}$ we have by the pushforward formula

$$
q_{2,1 ;+*}\left(\left(q_{2,1 ;+}^{*} f\right) \mu\right)=f q_{2,1 ;+*} \mu
$$

for any function $f$ on $\mathcal{S M}_{2}$. Extending to the compactification leads to an integration relation:

$$
\int_{\overline{\mathfrak{M}}_{2,1 ;+}}\left(q_{2,1 ;+}^{*} f\right) \mu=\int_{\overline{\mathcal{S M}_{2}} ;+} f q_{2,1 ;+*} \mu
$$

where now $f$ must be compactly supported. Note that since the fiber of $q_{2,1 ;+}$ is $(1 \mid 1)$ dimensional, i.e., not purely odd, the pushforward $q_{2,1 ;+*}$ will not coincide with the familiar Berezin-integration. Indeed, Berezin integration will reduce measures on $\mathfrak{M}_{2,1 ;+}$ to $\mathcal{S M}_{2,1 ;+}$ along the meromorphic projection $\pi_{2,1 ;+}: \mathfrak{M}_{2,1 ;+} \rightarrow \mathcal{S M}_{2,1 ;+}$. As a result, the Berezin integration of $\mu$ along $\pi_{2,1}$ will introduce singularities in the pushed-forward measure $\pi_{2,1 ;+*} \mu$.

The integration formula in (3.16) involves the compactifications of supermoduli space and spin moduli space. Witten in [8, Sec. 5], observes however that the D'Hoker-Phong projection $\pi_{2 ;+}: \mathfrak{M}_{2 ;+} \rightarrow \mathcal{S M}_{2 ;+}$ does not extend to a holomorphic projection of the compactification $\overline{\mathfrak{M}}_{2 ;+}$. Indeed, along the divisor $\mathcal{D}_{N S ; s e p . ;-}$ parametrising separating NS degenerations of type $(-,-), \pi_{2 ;+}$ fails to both $(1)$ be holomorphic along $\mathcal{D}_{N S ; s e p ;-;}$; and (2) to project $\mathcal{D}_{N S ; \text { sep.;- }}$ onto its reduced space. As a result, the extension to the boundary is generally meromorphic, so there exists a commutative diagram:

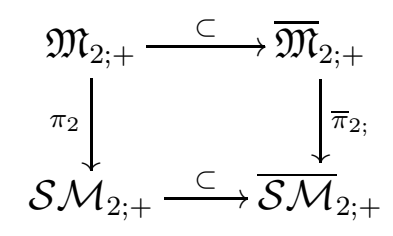

where $\pi_{2 ;+}$ is the holomorphic D'Hoker-Phong projection, and $\bar{\pi}_{2 ;+}$ is a meromorphic extension to the compactification. There are however boundary components along which $\pi_{2}$ will extend holomorphically. One such boundary component is the divisor $\mathcal{D}_{N S ; \text { sep.; }}$ parametrising separating NS degenerations of type $(+,+)$. It was noted by Witten in 
[8, Sec. 5], that $\bar{\pi}_{2 ;+}$ will both (1) be holomorphic along $\mathcal{D}_{N S ; s e p ;+}$; and (2) project $\mathcal{D}_{N S ; s e p . ;+}$ onto its reduced space. The relation in (3.16) will therefore be valid near the divisor $\mathcal{D}_{N S ; s e . ;+}$.

Now recall that the vacuum amplitude at $g$-loop order is obtained by integration over $\overline{\mathfrak{M}}_{g}$. A partial $g$-loop boundary contribution is then a contribution from the factor $\overline{\mathfrak{M}}_{2,1}$ in the boundary. As explained above however, the holomorphic, D'Hoker-Phong projection $\pi_{2 ;+}: \mathfrak{M}_{2 ;+} \rightarrow \mathcal{S M}_{2 ;+}$ will extend generally to a meromorphic projection upon full compactification; and as noted in the comments succeeding (3.17), $\bar{\pi}_{2 ;+}$ will be holomorphic along the NS separating divisor $\mathcal{D}_{N S ; s e p . ;+}$. Hence we can look at the 'partial' compactification

$$
\overline{\mathfrak{M}}_{g ; N \text {; sep. }}:=\mathfrak{M}_{g} \cup \mathcal{D}_{N S ; \text { sep. }} \subset \overline{\mathfrak{M}}_{g},
$$

where super Riemann surfaces are only allowed to develop NS-nodes. For the even part we have,

$$
\overline{\mathfrak{M}}_{2 ; N S ; s e p ;++}:=\mathfrak{M}_{2 ;+} \cup \mathcal{D}_{N S ; s e p ; ;+} \subset \overline{\mathfrak{M}}_{2 ;+} .
$$

The projection map $\overline{\mathfrak{M}}_{2 ; N \text { S;sep.;+ }} \stackrel{\bar{\pi}_{2 ;+}}{\longrightarrow} \overline{\mathcal{S M}}_{2 ;+}$, is holomorphic.

More generally now, for any superspace $Y$ with integration measure $\mu_{Y}$ and holomorphic map

$$
\rho: Y \rightarrow \overline{\mathfrak{M}}_{2 ;+},
$$

we can integrate $\rho_{*} \mu_{Y}$ along the partial compactification $\overline{\mathfrak{M}}_{2 ; N S ; s e p ; ;+}$ as in (3.16). The integral of superstring measures along this partial compactification will be referred to as a partial $g$-loop contribution by using the genus two supermoduli space as a building block, or factor.

The boundary of $\overline{\mathfrak{M}}_{g}$, denoted $\partial \overline{\mathfrak{M}}_{g}$, has codimension $(1 \mid 0)$. This means the normal bundle $\nu_{g}$ to the embedding $\partial \overline{\mathfrak{M}}_{g} \subset \overline{\mathfrak{M}}_{g}$ has rank (1|0) and can locally be parametrised by one even variable. The conormal bundle sequence to this embedding is

$$
\left.0 \longrightarrow \nu_{g}^{*} \longrightarrow \Omega_{\bar{M}_{g}}^{1}\right|_{\partial \overline{\mathfrak{M}}_{g}} \longrightarrow \Omega_{\partial \overline{\mathfrak{M}}_{g}}^{1} \longrightarrow 0 .
$$

Taking the Berezinian of (3.21) and using that $\nu_{g}^{*}$ has rank-(1|0) therefore gives the isomorphism

$$
\left.\operatorname{Ber} \overline{\mathfrak{M}}_{g}\right|_{\partial \overline{\mathfrak{M}}_{g}} \cong \nu_{g}^{*} \otimes \text { Ber } \partial \overline{\mathfrak{M}}_{g} \text {. }
$$

The isomorphism (3.22) relates measures on $\overline{\mathfrak{M}}_{g}$ with measures on the boundary $\partial \overline{\mathfrak{M}}_{g}$. With the characterisation of the boundary components in (2.4) and (2.6), the isomorphism in (3.22) leads to ansatz factorisations for the superstring measure near specified boundary components, e.g., Ber $\left.\partial \overline{\mathfrak{M}}_{g}\right|_{\Delta_{g_{1}, g-g_{1}}} \cong \operatorname{Ber} \overline{\mathfrak{M}}_{g_{1}, 1} \otimes \operatorname{Ber} \overline{\mathfrak{M}}_{g-g_{1}, 1}$.

The configuration space for the superstring vacuum states at $g$-loop order is $\overline{\mathfrak{M}}_{g}$. Along a NS separating divisor $\mathcal{D}_{N S ; s e p .} \subset \partial \overline{\mathfrak{M}}_{g}$ with generic component in (2.4), observe that the number of punctures of each factor will always satisfy the inequality (3.13) when 


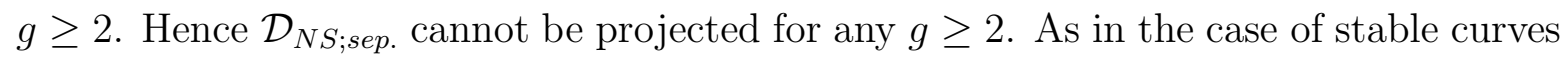
however, the forgetful map from punctured supermoduli space $\overline{\mathfrak{M}}_{g, 1} \stackrel{p}{\rightarrow} \overline{\mathfrak{M}}_{g}$ realises $\overline{\mathfrak{M}}_{g, 1}$ as the universal family of genus $g$, super Riemann surfaces over $\overline{\mathfrak{M}}_{g}$. And so, forgetting the puncture yields a morphism $\mathcal{D}_{N S, s e p .} \cong \overline{\mathfrak{M}}_{g_{1}, 1} \times \overline{\mathfrak{M}}_{g_{2}, 1} \rightarrow \overline{\mathfrak{M}}_{g_{1}} \times \overline{\mathfrak{M}}_{g_{2}}$, where $g_{1}+g_{2}=g$. If either of the factors $\overline{\mathfrak{M}}_{g_{1}}$ or $\overline{\mathfrak{M}}_{g_{2}}$ are projected, then measures on $\mathcal{D}_{N S \text {,sep. }}$ can be pushed-forward and integrated over the projected factor $\overline{\mathfrak{M}}_{g_{1}}$ or $\overline{\mathfrak{M}}_{g_{2}}$. In this way, components of the integration measure over $\mathcal{D}_{N S \text {,sep. }}$ can be calculated by integrating along the composite morphism

$$
\mathcal{D}_{N S, \text { sep. }} \cong \overline{\mathfrak{M}}_{g_{1}, 1} \times \overline{\mathfrak{M}}_{g_{2}, 1} \rightarrow \overline{\mathfrak{M}}_{g_{1}} \quad \text { or } \quad \mathcal{D}_{N S, \text { sep. }} \cong \overline{\mathfrak{M}}_{g_{1}, 1} \times \overline{\mathfrak{M}}_{g_{2}, 1} \rightarrow \overline{\mathfrak{M}}_{g_{2}}
$$

Now recall the morphism $q_{2,1 ;+}$ from (3.14). Extending it to the compactification results in a map,

$$
\bar{q}_{2,1 ;+}: \overline{\mathfrak{M}}_{2,1 ;+} \stackrel{p}{\longrightarrow} \overline{\mathfrak{M}}_{2 ;+} \stackrel{\bar{\pi}_{2 ;+}}{\longrightarrow} \overline{\mathcal{S M}}_{2 ;+} .
$$

Specialising $\bar{q}_{2,1 ;+}$ to the partial compactification along $(+,+)$-separating NS nodes in (3.19) then gives a holomorphic map. In genus $g \geq 2$ now, setting $g_{1}=2$ so that $g_{2}=g-2$ gives the boundary component:

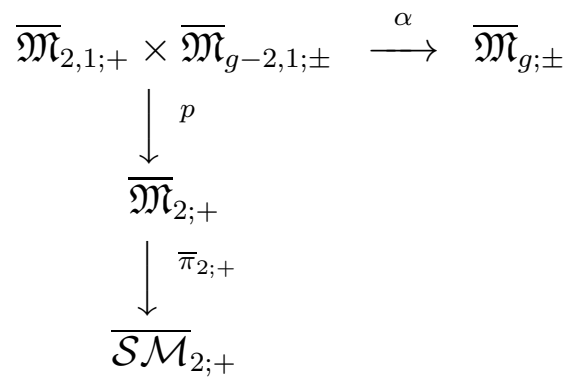

Note that the above diagram is compatible with both even and odd components of $\overline{\mathfrak{M}}_{g}$ and $\overline{\mathfrak{M}}_{g-2,1}$ respectively. In this way we obtain a holomorphic map $\mathcal{D}_{N S ; s e p .} \rightarrow \overline{\mathfrak{M}}_{2 ;+}$.

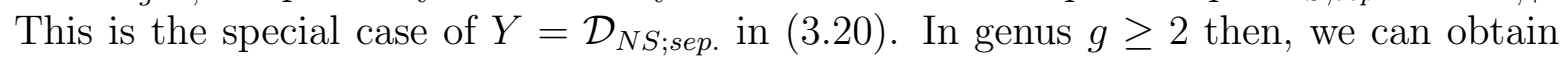
a partial, $g$-loop vacuum contribution from the boundary component $\mathcal{D}_{N S ; \text { sep. }}$. In the case of a non-separating, NS degeneration $\mathcal{D}_{N S ; n s e p .} \subset \partial \overline{\mathfrak{M}}_{g}$, recall from (2.4) that $\mathcal{D}_{N S ; n s e p .} \cong \overline{\mathfrak{M}}_{g-1,2}$. The inequality in (3.13) holds for genus $g \geq 4$; while in genus $g=3$, (3.13) does not hold. Nevertheless, in genus $g=3$, projecting out the two NS-punctures yields:

$$
\mathcal{D}_{\text {NS;nsep.;+ }} \stackrel{\cong}{\longrightarrow} \overline{\mathfrak{M}}_{2,2 ;+} \stackrel{p^{2}}{\longrightarrow} \overline{\mathfrak{M}}_{2 ;+} .
$$

As in the general case in (3.25) then, we can obtain a partial, three-loop boundary contribution from the non-separating divisor $\mathcal{D}_{N S ; n s e p . ;+}$ to the three-loop superstring vacuum amplitude.

Recall from (2.6) that the divisors parametrising degenerations along Ramond punctures are fibered over supermoduli spaces with (0|1)-dimensional fiber. The key difference here between calculating partial three loop contributions from these boundary 
components lies therefore in firstly integrating out the odd fiber parameter along the given fibrations $\pi_{R ; s e p . ;+}$ and $\pi_{R ; n s e p . ;+}$ in (2.6) respectively. This allows for reducing the integration measure to measures on supermoduli spaces. An analysis similar to the case of NS-degenerations can be undertaken to evaluate partial three-loop contributions along the divisors parametrising degenerations along Ramond punctures.

The below diagram is another example for a higher genus, namely $g=4$ :

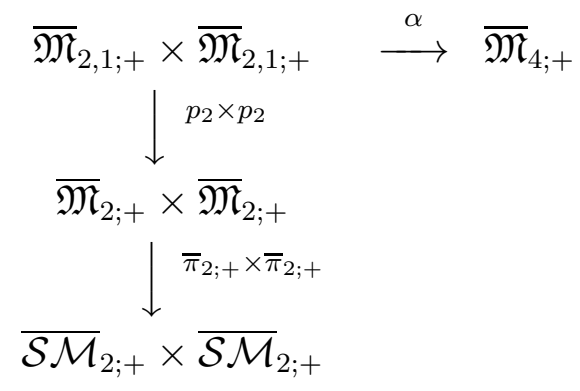

We will illustrate the example of $g=3$ in Section 3.5. In some special cases in the context of $g=3,4$, both factors could be projectable as in above diagram (3.27).

\subsection{Degenerations and super Mumford forms}

In analogy with the Mumford relations on the moduli space of Riemann surfaces, there are analogous relations between divisors on the supermoduli space, as illustrated in (3.12). Generalising these relations to the punctured case leads to a trivialising section of a certain tensor product involving canonical bundles, $\Psi_{g, n, n^{\prime}}$ on $\overline{\mathfrak{M}}_{g, n, n^{\prime}}$, referred to as the super Mumford form. This global section $\Psi_{g, n, n^{\prime}}$ defines a holomorphic measure on $\overline{\mathfrak{M}}_{g, n, n^{\prime}}$ and, in perturbative superstring theory, is taken to be the $g$ loop, superstring measure with $n+n^{\prime}$ external states. Its integral over $\overline{\mathfrak{M}}_{g, n, n^{\prime}}$ gives the $g$ loop scattering amplitude. There are a number of issues surrounding the computation of this amplitude however, arising primarily from the complexity in the geometry of the supermoduli space itself.

For instance, $\Psi_{g, 0}$ corresponds to vacuum amplitudes where there are no external particles; and $\Psi_{g, 1}$ corresponds to tadpole amplitudes or one-point amplitudes. The form $\Psi_{g, 2}$ corresponds to two-point amplitudes or propagators of a string state. The tadpole graphs are those with only one external state or external particle. The tadpole amplitudes describe the amplitudes for a particle to disappear into the vacuum. In unitary superstring theory, the tadpole amplitude for a massless stable particle is zero.

An attractive feature of looking at the boundary of supermoduli space is that, as is clear from (2.4), (2.6), its components parametrise super Riemann surfaces of lower genera. Furthermore, near the boundary, the superstring measure itself factorises into lower genus components, c.f., the discussions around (3.22). In genus $g=g_{1}+g_{2}$, we 
have the following $\alpha$ - and $\beta$-type clutchings such as

$$
\overline{\mathfrak{M}}_{g_{1}, n_{1}+1, n_{1}^{\prime}} \times \overline{\mathfrak{M}}_{g_{2,}, n_{2}+1, n_{2}^{\prime}} \stackrel{\alpha}{\longrightarrow} \overline{\mathfrak{M}}_{g, n, n^{\prime}}, \quad \overline{\mathfrak{M}}_{g-1, n+2, n^{\prime}} \stackrel{\beta}{\longrightarrow} \overline{\mathfrak{M}}_{g, n, n^{\prime}} .
$$

The images of $\alpha$ and $\beta$ form components of the boundary $\partial \overline{\mathfrak{M}}_{g, n, n^{\prime}}$ which we denote $\Delta_{\alpha}$ and $\Delta_{\beta}$ respectively. We use the geometric model of gluing maps to analyse boundary contributions, via observations by Witten [7, 8]. Along a separating or non-separating degeneration respectively, with $g=g_{1}+g_{2}$, the super Mumford form satisfies the ansatz factorisation

$$
\left.\Psi_{g, n, n^{\prime}}\right|_{\Delta_{\alpha}} \sim \Psi_{g_{1}, n_{1}+1, n_{1}^{\prime}} F(\varepsilon) \Psi_{g_{2}, n_{2}+1, n_{2}^{\prime}},\left.\quad \Psi_{g, n, n^{\prime}}\right|_{\Delta_{\beta}} \sim \Psi_{g-1, n+2, n^{\prime}} G(\varepsilon),
$$

for some possible singular forms $F(\varepsilon)$ and $G(\varepsilon)$ depending on the degeneration parameter $\varepsilon$. The tilde sign $\sim$ means up to a multiplicative constant, which is a normalisation constant in the definition of $\Psi$. By (3.29) then, the super Mumford form is expected to satisfy relevant asymptotics near the boundary divisors. Integrating these asymptotic expressions for $\Psi_{g, n, n^{\prime}}$ along the boundary reveals how the $g$-loop amplitude will receive contributions from the boundary of $\overline{\mathfrak{M}}_{g, n, n^{\prime}}$.

Away from the boundary divisor, the node is smoothed out into a thin cylinder. The closer to the boundary divisor in the supermoduli space, the thinner the cylinder is. The thin cylinder means a long distance propagation of a closed string state in string theory. From the worldsheet superconformal field theory point of view, gluing along the NS punctures as in (2.15) is equivalent to insertion of the form $V_{\ell}(a \mid \alpha) \otimes V_{r}(b \mid \beta) \varepsilon^{m} \mathrm{~d} \varepsilon$, where $\varepsilon$ is the gluing parameter and $V_{\ell}$ and $V_{r}$ are operators inserted on the two sides of the cylinder at points $a \mid \alpha$ and $b \mid \beta$. The conformal dimension of the operator is $1+\frac{m}{2}$ for $m \geq-2$.

The forms $F(\varepsilon)$ and $G(\varepsilon)$ are defined on the cylinder described by the degeneration parameter $\varepsilon$. A form with a general order $m$ is given by the expression

$$
[\mathrm{d} a \mid \mathrm{d} \alpha] \varepsilon^{m} \mathrm{~d} \varepsilon[\mathrm{d} b \mid \mathrm{d} \beta] .
$$

In the gluing relations (2.15), notice that $\varepsilon$ is related to the square-root of the NS gluing parameter $q_{N S}$. To investigate this relation further, we can consider a more general change of variable from $\varepsilon$ to a new gluing parameter $q$ as follows,

$$
(-q)^{1 / 2}=\varepsilon+\varepsilon^{p} C \alpha \beta
$$

or equivalently $q=-\varepsilon\left(\varepsilon+2 C \varepsilon^{p} \alpha \beta\right)$ with $p$ a general real number. Integrating over the odd moduli $\alpha$ and $\beta$ while fixing $q$ then yields,

$$
\begin{aligned}
\int[\mathrm{d} a \mid \mathrm{d} \alpha] \varepsilon^{m} \mathrm{~d} \varepsilon[\mathrm{d} b \mid \mathrm{d} \beta] & \sim \int[\mathrm{d} \alpha]\left(\left(\mathrm{d} a C q^{\frac{1}{2}(m+p-2)} \mathrm{d} q \mathrm{~d} b\right) \alpha \beta+\ldots\right)[\mathrm{d} \beta] \\
& \sim \int \mathrm{d} a C q^{\frac{1}{2}(m+p-2)} \mathrm{d} q \mathrm{~d} b .
\end{aligned}
$$


Here $\mathrm{d} a, \mathrm{~d} q, \mathrm{~d} b$ are independent integration variables. Accordingly, near a boundary component $\Delta_{j}$ the super Mumford form factorises as follows,

$$
\left.\Psi_{g, n, n^{\prime}}\right|_{\Delta_{j}} \sim \Psi_{\Delta_{j}} \frac{\mathrm{d} q}{q^{\frac{1}{2}(2-m-p)}}
$$

where $\Psi_{\Delta_{j}} \in \Gamma\left(\operatorname{Ber} T_{\Delta_{j}}^{*} \otimes\left(\operatorname{Ber} T_{\Delta_{j}}\right)^{5}\right)$ and $\Psi_{g, n, n^{\prime}} \in \Gamma\left(\operatorname{Ber} T_{\mathfrak{M}_{g, n, n^{\prime}}}^{*} \otimes\left(\operatorname{Ber} T_{\overline{\mathfrak{M}}_{g, n, n^{\prime}}}\right)^{5}\right)$ has a pole of a certain order on the boundary $\Delta_{j} \subset \overline{\mathfrak{M}}_{g, n, n^{\prime}}$. This formula (3.33) is derived from the gluing map of the geometric model by analytic methods, and is also a realisation of the factorisation in (3.22). The integration on the reduced space of the cylinder is

$$
\int \frac{\mathrm{d}^{2} q}{q^{\frac{1}{2}(2-m-p)} \bar{q}^{\frac{1}{2}(2-m-p)}}
$$

which would have a pole for $m+p<2$. This physically means a propagator of a closed string state on a cylinder. In fact, the Neveu-Schwarz (or Ramond) node is the limit when the circle winding the cylinder, for a propagating Neveu-Schwarz state (or Ramond state), is shrinking to zero size.

In the case where the boundary divisor parametrises separating NS degenerations of $(+,+)$-type, Witten [7, 18] derived,

$$
\Psi_{g ; \pm} \sim \Psi_{g_{1} ;+}[\mathrm{d} a \mid \mathrm{d} \alpha] \frac{\mathrm{d} \varepsilon}{\varepsilon^{2}}[\mathrm{~d} b \mid \mathrm{d} \beta] \Psi_{g_{2} ; \pm} .
$$

This corresponds to $m=-2, p=0$. Note that there is always a $\Psi_{g_{1} ;+}$ factor with + spin structure in the above factorisation. At a $(-,-)$ degeneration [7, 18],

$$
\Psi_{g ;+} \sim \Psi_{g_{1 ;-}}(V)[\mathrm{d} a \mid \mathrm{d} \alpha] \mathrm{d} \varepsilon \varepsilon^{8}[\mathrm{~d} b \mid \mathrm{d} \beta] \Psi_{g_{2 ;}-}(V),
$$

where $\Psi_{g_{i} ;-}(V)$ is computed by inserting the superconformal primary operator $V$ of dimension 5 , and $m=8, p=-2$. Note that this term scales as a positive power of $\varepsilon$. After change of variable, this term scales as a positive power of $q$. Hence the integration of this term (3.36) is vanishing in the limit of $\varepsilon \rightarrow 0$ or $q \rightarrow 0$, and so the contribution to the $g$-loop amplitude from this boundary component is vanishing.

For non-separating degeneration of NS type we have the factorisation,

$$
\Psi_{g} \sim \Psi_{g-1}[\mathrm{~d} a \mid \mathrm{d} \alpha] \frac{\mathrm{d} \varepsilon}{\varepsilon^{2}}[\mathrm{~d} b \mid \mathrm{d} \beta] .
$$

Changing variable from $\varepsilon$ to $q$ via $(-q)^{1 / 2}=\varepsilon+\alpha \beta$ [7] and integrating over $\alpha$ and $\beta$ with fixed $q$ gives,

$$
\int[\mathrm{d} \alpha] \varepsilon^{-2} \mathrm{~d} \varepsilon[\mathrm{d} \beta] \sim \int[\mathrm{d} \alpha]\left(q^{-2} \mathrm{~d} q \alpha \beta+\ldots\right)[\mathrm{d} \beta]=\int q^{-2} \mathrm{~d} q,
$$


and hence:

$$
\begin{aligned}
\left.\pi_{*} \Psi_{g}\right|_{\operatorname{im} \alpha_{g_{1}, g_{2}}} & \sim \Psi_{g_{1}}^{\mathrm{d} a} \frac{\mathrm{d} q}{q^{2}} \Psi_{g_{2}}^{\mathrm{d} b} \\
\left.\pi_{*} \Psi_{g}\right|_{\operatorname{im} \beta_{g-1}} & \sim \Psi_{g-1}^{\mathrm{d} a \mathrm{~d} b} \frac{\mathrm{d} q}{q^{2}}
\end{aligned}
$$

Here $\pi_{*}$ denotes integrating out two fermionic moduli e.g. $\mathrm{d} \alpha \mathrm{d} \beta$. Furthermore, we have used forgetful morphsims in defining the forms appearing on the right hand side above, e.g., $\Psi_{g_{1}, 1}=p^{*}\left(\Psi_{g_{1}}\right)$. The form $\Psi_{g_{1}}^{\mathrm{d} a}$ is then the contraction of $\Psi_{g_{1}}$ along da. Eq. (3.39), like (3.32), contains factors like $\mathrm{d} a \mathrm{~d} q \mathrm{~d} b$ with independent variables for integration.

From the worldsheet superconformal field theory point of view [7, 18], gluing along the Ramond punctures as in (2.16) is equivalent to insertions of the form

$$
e^{\zeta G_{0}}[\mathrm{~d} \zeta] \frac{\mathrm{d} q_{R}}{q_{R}},
$$

where $q_{R}$ and $\zeta$ are bosonic and fermionic gluing parameters and $G_{0}$ is the zero-mode of the worldsheet supercurrent. The factor $e^{\zeta G_{0}}$ is due to the coupling of the worldsheet gravitino with the worldsheet supercurrent in the action. The $\mathrm{d} \zeta$ is integrated over $\mathbb{C}^{0 \mid 1}$. From the point of view of the boundary divisor, the $\mathbb{C}^{0 \mid 1}$ is the extra fermionic fiber of the boundary divisor with Ramond degeneration in the supermoduli space.

For non-separating degeneration of Ramond type,

$$
\left.\Psi_{g}\right|_{\operatorname{im} \beta_{g-1}} \sim \sum_{\alpha_{i}, \alpha_{j}} \Psi_{g-1,2^{\prime}}\left(\Xi_{\alpha_{i}}, \Xi_{\alpha_{j}}\right) \frac{\mathrm{d} q_{R}}{q_{R}} e^{\zeta G_{0}}[\mathrm{~d} \zeta]
$$

Here $\Psi_{g-1 ; 2^{\prime}}\left(\Xi_{\alpha_{1}}, \Xi_{\alpha_{2}}\right)$ is the super Mumford form for a genus $g-1$ super Riemann surfaces with $n^{\prime}=2$ Ramond punctures and with the superconformal primary operators $\Xi_{\alpha_{1}}, \Xi_{\alpha_{2}}$ inserted at these punctures [8]. Recall that in the notation we use primes to denote the Ramond puncture number.

For separating degenerations of Ramond type near the boundary of the supermoduli space,

$$
\left.\Psi_{g, 2^{\prime}}\left(\Xi_{\alpha_{1}}, \Xi_{\alpha_{2}}\right)\right|_{\text {im } \alpha_{g_{1}, g_{2}}} \sim \sum_{\alpha_{i}, \alpha_{j}} \Psi_{g_{1}, 2^{\prime}}\left(\Xi_{\alpha_{1}}, \Xi_{\alpha_{i}}\right) \frac{\mathrm{d} q_{R}}{q_{R}} e^{\zeta G_{0}}[\mathrm{~d} \zeta] \Psi_{g_{2}, 2^{\prime}}\left(\Xi_{\alpha_{2}}, \Xi_{\alpha_{j}}\right),
$$

where $g_{1}+g_{2}=g$. Note that (3.43) is for the case when there are two external massless string states on the left-hand side of (3.43).

The factorisation is valid near the degeneration, when $|\varepsilon|$ or $\left|q_{R}\right|$ are small respectively, and the degeneration is the limit when $\varepsilon \rightarrow 0$ or $q_{R} \rightarrow 0$ respectively. 


\subsection{Integration in supergeometry and supermoduli spaces}

To calculate the superstring amplitude, integration on the supermoduli space is needed. This would involve firstly integrating out the odd (fermionic) coordinates, and then integrating the resulting measure over a classical moduli space. In this section, we first describe integration of holomorphic measures. We then describe integration over smooth supermanifolds and over complex supermanifolds. Then we present a useful integration formula (3.53) which will be used in Section 3.5. Finally, we describe integral forms which are also relevant to the question of integrating along the boundary of the supermoduli space.

On smooth, real, orientable manifolds $M$ there is, up to a positive constant, a natural and unique volume measure $\nu_{M}$. This $\nu_{M}$ is a global section of the line bundle of volume forms

$$
\operatorname{det} T^{*} M=\Omega^{\operatorname{dim} M}(M),
$$

where $\Omega^{1}(M)=\Gamma\left(M, T^{*} M\right)$ are the global sections of the cotangent vector bundle over $M$. Now we describe integration of holomorphic measures. Complex manifolds $X$ are smooth manifolds equipped with a choice of integrable complex structure. Any $n$ dimensional complex manifold $X$ will have an underlying $2 n$-dimensional real manifold which we denote by $X^{\infty}$. With $J$ an integrable, complex structure we can identify $X=\left(X^{\infty}, J\right)$. Volume forms on $X$ can be integrated over $M=X^{\infty}$ as a smooth real manifold. We want to describe holomorphic volume measures however which come from the complex manifold $X$. This requires understanding the decomposition of the differential forms on $X^{\infty}$. Let $T X^{\infty *}$ denote the cotangent bundle. With respect to the complex structure $J$ we have a decomposition into holomorphic and anti-holomorphic forms $T X^{\infty *} \cong T^{1,0} X^{\infty *} \oplus T^{0,1} X^{\infty *}$ and hence a tensor product factorisation of the volume forms on $X^{\infty}$. Now with $T^{*} X$ the holomorphic cotangent bundle of $X$ there is a natural inclusion $T^{*} X \subset T^{1,0} X^{\infty *}$; and similarly an inclusion of the anti-holomorphic cotangent bundle $\overline{T^{*} X} \subset T^{0,1} X^{\infty *}$. The inclusions are at the level of the sections of vector bundles. Denote $\operatorname{det} X=\operatorname{det} T^{*} X$ and $\operatorname{det} \bar{X}=\operatorname{det} \overline{T^{*} X}$. Using (3.44) we have therefore an inclusion into the volume forms on $X^{\infty}$,

$$
\operatorname{det} X \otimes \operatorname{det} \bar{X} \subset \operatorname{det} T^{1,0} X^{\infty *} \otimes \operatorname{det} T^{0,1} X^{\infty *} \cong \operatorname{det} X^{\infty} .
$$

Sections of $\operatorname{det} X$ are referred to as holomorphic volume forms on $X$; and similarly sections of $\operatorname{det} \bar{X}$ are anti-holomorphic volume forms. Complex conjugation $z \mapsto \bar{z}$ induces a conjugation on holomorphic forms. In particular, to any holomorphic volume form $\omega \in \operatorname{det} X$ we have the conjugate-squaring

$$
\operatorname{det} X \longrightarrow \operatorname{det} X \otimes \operatorname{det} \bar{X}, \quad \omega \longmapsto \omega \otimes \bar{\omega} .
$$

Composing (3.45) with (3.46) gives a mapping between volume forms $\operatorname{det} X \rightarrow \operatorname{det} X^{\infty}$. And so, with this mapping, we can integrate holomorphic functions against holomorphic 
volume forms on $X$ by simply integrating the resulting $(n, n)$-form over the underlying real manifold $X^{\infty}$. Explicitly, for a holomorphic volume form $\omega$ on a complex manifold $X$, we have

$$
\int_{X} \omega \stackrel{\text { def }}{=} \int_{X^{\infty}} \omega \otimes \bar{\omega}
$$

Before turning to integration in supergeometry, we discuss a notion in algebraic topology serving to motivate subsequent notions in supergeometry. A vector bundle $E$ over a manifold $M$ is fibered over $M$ with linear fibers. With $\pi: E \rightarrow M$ denoting the fibration, compactly supported forms on $E$ can be formally reduced to forms on $M$ via the integration-along-fiber map $\pi_{*}: \Omega_{c p c t .}^{j}(E) \rightarrow \Omega^{j-\text { rank } E}(M)$. As explained by Bott and $\mathrm{Tu}$ [27], if $x$ denote local coordinates on the base $M$ and $y$ coordinates on the fiber, then $(x, y)$ will be local coordinates on $E$ and $\pi_{*}$ is defined on $\Omega_{c p c t .}^{j}(E)$ by:

$$
\pi_{*}:\left(\pi^{*} f\right) \mathrm{d} x_{1} \cdots \mathrm{d} x_{m} \mathrm{~d} y_{1} \cdots \mathrm{d} y_{m^{\prime}} \longmapsto \begin{cases}f(x) \mathrm{d} x_{1} \cdots \mathrm{d} x_{j-\text { rank } E} & \text { if } m^{\prime}=\operatorname{rank} E \\ 0 & \text { otherwise. }\end{cases}
$$

Intuitively, that $\pi_{*}\left(\mathrm{~d} y_{1} \cdots \mathrm{d} y_{m^{\prime}}\right)=1$ if $m^{\prime}=\operatorname{rank} E$ and is zero otherwise. Berezin [1] defined integration over supermanifolds analogously to the integration-along-fiber map above. Crucially, this definition only makes sense if the supermanifold can be fibered over its reduced space with purely odd fibers - a property known as 'projected'. That any supermanifold can be smoothly projected over its reduced space is a consequence of Batchelor's splitting theorem [28]. Holomorphically however, there are generally obstructions to fibering (or, projecting) complex supermanifolds over their reduced spaces. Hence, holomorphic measures over complex supermanifolds cannot generally be integrated in the way outlined by Berezin. In the case where complex supermanifolds $\mathfrak{X}$ are non-projected, i.e., cannot be holomorphically fibered over their reduced space, it is an open question as to how to integrate holomorphic measures over $\mathfrak{X}$. Donagi and Witten [19, 22] found that $\mathfrak{M}_{g}$ cannot be globally holomorphically projected onto its reduced space for any genus greater or equal to five.

To continue our discussion of the integration on supermanifolds now, there are two kinds of objects which can be integrated over supermanifolds. They are (1) Berezinian volume forms; and (2) integral forms. The former are similar to volume forms on manifolds as discussed above; and the latter are similar to distributions. We will firstly consider Berezinian volume forms.

There is no unique 'top form' on a supermanifold since the differential of odd, or Grassmann, variables are no longer nilpotent. For example, if $\theta$ is odd, then $\mathrm{d} \theta \wedge \mathrm{d} \theta \neq 0$. One can nevertheless form the module of volume forms on a supermanifold analogous to the determinant line bundle from (3.44). To any super vector space $\mathbb{V}=V \oplus \Pi W$, where $\Pi W$ is the vector space of Grassmann variables with fermionic statistics, we can form its Berezinian Ber $\mathbb{V}$, which is a (1|1)-dimensional vector space. Now for a supermanifold $\mathfrak{X}$ its cotangent bundle $T^{*} \mathfrak{X}$ is is a bundle of super vector spaces. It makes sense to 
therefore set Ber $\mathfrak{X}=\operatorname{Ber} T^{*} \mathfrak{X}$. To see how to integrate these volume forms, let $|\mathfrak{X}|$ be the reduced space of $\mathfrak{X}$. It is smooth manifold and embeds naturally inside $\mathfrak{X}$. With a projection map $\pi: \mathfrak{X} \rightarrow|\mathfrak{X}|$, the supermanifold $\mathfrak{X}$ can be realised as fibered over its reduced space with odd or 'fermionic fiber'. Generalising the classical integration-alongfiber construction in differential topology, we can use $\pi$ to integrate out the fermionic fibers to recover thereby a volume form on $|\mathfrak{X}|$. Denoting by $\pi_{*}$ the integration-alongfiber map, we have therefore a morphism of sheaves $\pi_{*}$ : Ber $\mathfrak{X} \rightarrow \operatorname{det}|\mathfrak{X}|$. With $\pi$ then we can define, for any $\sigma_{\mathfrak{X}} \in \Gamma(\mathfrak{X}, \operatorname{Ber} \mathfrak{X})$ :

$$
\int_{\mathfrak{X}} \sigma_{\mathfrak{X}} \stackrel{\text { def }}{=} \int_{|\mathfrak{X}|} \pi_{*} \sigma_{\mathfrak{X}} .
$$

Now suppose $\mathfrak{X}$ is endowed with a covering $\mathfrak{U}=\left(\mathfrak{U}_{\alpha}\right)$ where each $\mathfrak{U}_{\alpha}$ is isomorphic to $\left(\left|\mathfrak{U}_{\alpha}\right|, C^{\infty}\left(\left|\mathfrak{U}_{\alpha}\right|\right) \otimes \wedge^{\bullet} \mathbb{R}^{q}\right)$ where $\left|\mathfrak{U}_{\alpha}\right|$ is the reduced space of $\mathfrak{U}_{\alpha}$. Denote the odd dimension of $\mathfrak{X}$ by $q$. Let $F \in \mathcal{O}_{\mathfrak{X}}(\mathfrak{X})$ be a global, smooth function. Then over $\mathfrak{U}_{\alpha}$ we have $\left.F\right|_{\mathfrak{U}_{\alpha}} \in \mathcal{O}_{\mathfrak{X}}\left(\mathfrak{U}_{\alpha}\right) \cong C^{\infty}\left(\left|\mathfrak{U}_{\alpha}\right|\right) \otimes \wedge^{\bullet} \mathbb{R}^{q}$. With the projection map $\pi: \mathfrak{X} \rightarrow|\mathfrak{X}|$, we can write $\left.F\right|_{\mathfrak{U}_{\alpha}}=\left(\pi^{*} g_{\alpha}\right) \otimes \Theta_{\alpha}$ for some $g_{\alpha} \in C^{\infty}\left(\left|\mathfrak{U}_{\alpha}\right|\right)$ and a Grassmann constant $\Theta_{\alpha} \in \wedge \bullet \mathbb{R}^{q}$. Note that this constant can be absorbed into the Berezinian volume form $\sigma_{\mathfrak{X}, \alpha}=\left.\sigma_{\mathfrak{X}}\right|_{\mathfrak{U}_{\alpha}}$. Finally now, in order to ensure the ultimate integral is well defined, choose a partition of unity $\rho_{|\mathfrak{U}|}$ subordinate to $|\mathfrak{U}|$ and set $f_{\alpha}=\rho_{\alpha} g_{\alpha}$. For each index $\alpha, \rho_{\alpha}$ is compactly supported in $\left|\mathfrak{U}_{\alpha}\right|$. Then over $\mathfrak{U}_{\alpha}$ we have by (3.49),

$$
\left.\int_{\mathfrak{U}_{\alpha}} F\right|_{\mathfrak{U}_{\alpha}} \sigma_{\alpha}=\int_{|\mathfrak{X}|} f_{\alpha} \pi_{*}\left(\Theta_{\alpha} \sigma_{\mathfrak{X}, \alpha}\right) .
$$

We can integrate against any volume form $\sigma \in \Gamma(\mathfrak{X}$, Ber $\mathfrak{X})$,

$$
\int_{\mathfrak{X}} F \sigma_{\mathfrak{X}}=\sum_{\alpha} \int_{\mathfrak{U}_{\alpha}} F_{\alpha} \sigma_{\mathfrak{X}, \alpha} .
$$

By (3.49), the integration above only depends on a choice of projection $\pi: \mathfrak{X} \rightarrow|\mathfrak{X}|$.

We now describe integration over complex supermanifolds. Firstly, there are a number of ways to define a complex supermanifold. For our purposes, a complex supermanifold $\mathfrak{Y}$ is a supermanifold where: (1) the reduced space $|\mathfrak{Y}|$ of $\mathfrak{Y}$ is a complex manifold and; (2) the restriction of the tangent bundle $T \mathfrak{Y}$ to $|\mathfrak{Y}|$ is holomorphic. The tangent bundle of $\mathfrak{Y}$ is $\mathbb{Z}_{2}$-graded, so $T \mathfrak{Y} \cong T_{+} \mathfrak{Y} \oplus T-\mathfrak{Y}$. Restricting $T \mathfrak{Y}$ to $|\mathfrak{Y}|$ gives $\left.T \mathfrak{Y}\right|_{|\mathfrak{Y}|} \cong T|\mathfrak{Y}| \oplus N|\mathfrak{Y}|$, where $N|\mathfrak{Y}| \rightarrow|\mathfrak{Y}|$ is a vector bundle. If $|\mathfrak{Y}|$ is a complex manifold, then $T|\mathfrak{Y}|$ will automatically be holomorphic. Condition (2) then amounts to requiring $N|\mathfrak{Y}|$ also be holomorphic. As in the case of complex manifolds, for complex supermanifolds there will be an underlying smooth supermanifold which we denote by $\mathfrak{Y}^{\infty}$. Our conventions here are such that the complex structure is only defined by reference to the even coordinates. And so, if $(z \mid \theta)$ denote local coordinates on $\mathfrak{Y}$, their 
conjugation is $\widetilde{(z \mid \theta)}=(\widetilde{z} \mid \theta)$. As explained by Witten in [31], the conjugate $\widetilde{z}$ coincides with the familiar complex conjugate $\bar{z}$ on the reduced space $\left|\mathfrak{Y}^{\infty}\right|$. That is, along the embedding $\left|\mathfrak{Y}^{\infty}\right| \subset \mathfrak{Y}^{\infty}$ we have $\widetilde{(z \mid 0)}=(\bar{z} \mid 0)$. As a consequence of this convention, the conjugate supermanifold $\widetilde{\mathfrak{Y}}$ only differs from $\mathfrak{Y}$ in that the reduced space is conjugate, i.e, $|\overline{\mathfrak{Y}}|=\overline{|\mathfrak{Y}|}$. Where the odd tangent bundle is concerned however, we have $T_{-} \widetilde{\mathfrak{Y}}=T_{-} \mathfrak{Y}$.

Now let $\pi: \mathfrak{Y} \rightarrow|\mathfrak{Y}|$ be a holomorphic projection. On Berezinian volume forms it defines the mapping, $(f+g \theta)[\mathrm{d} x \mid \mathrm{d} \theta] \stackrel{\pi_{*}}{\longmapsto} g d x$, for $x$ denoting a complex, even variable $x$ and $\theta$ the odd variable. For multiple even variables $x_{1}, \ldots, x_{m}$ and odd variables $\theta_{1}, \ldots, \theta_{n}$,

$$
\theta_{1} \cdots \theta_{n}\left[\mathrm{~d} x_{1} \cdots \mathrm{d} x_{m} \mid \mathrm{d} \theta_{1} \cdots \mathrm{d} \theta_{n}\right] \stackrel{\pi_{*}}{\longmapsto} \mathrm{d} x_{1} \cdots \mathrm{d} x_{m}
$$

Then as in the smooth case, $\pi$ realises $\mathfrak{Y}$ as being holomorphically fibered over its reduced space with fermionic fibers. With Ber $\mathfrak{Y}$ the space of holomorphic volume forms on $\mathfrak{Y}$, and $\operatorname{det}|\mathfrak{Y}|$ the holomorphic volume forms on the reduced space $|\mathfrak{Y}|$, the integration-along-fiber map gives a relation $\pi_{*}$ : Ber $\mathfrak{Y} \rightarrow \operatorname{det}|\mathfrak{Y}|$. Recall that by our conventions here, we only conjugate the even parameters. As such, and since the even and odd parameters are globally distinguished on $\mathfrak{Y}$, there is no need to implement an operation as in (3.46) for volume forms on $\mathfrak{Y}$ directly. We can instead defer this operation to the reduced space $|\mathfrak{Y}|$. Therefore, for a volume form $\Psi \in \Gamma(\mathfrak{Y}$, Ber $\mathfrak{Y})$ and a holomorphic projection $\pi: \mathfrak{Y} \rightarrow|\mathfrak{Y}|$, we can define:

$$
\int_{\mathfrak{Y}} \Psi \stackrel{\text { def }}{=} \int_{|\mathfrak{Y}|} \pi_{*} \Psi \otimes \overline{\pi_{*} \Psi} .
$$

Note that the right-hand side above does not make any reference to the underlying, smooth supermanifold $\mathfrak{Y}^{\infty}$. The procedure for calculating $\pi_{*} \Psi$ however is similar to that for volume forms on smooth supermanifolds $\mathfrak{X}$ since $\pi$ here is holomorphic. This can be viewed as being in analogy with the integration of holomorphic volume forms in (3.47). In Section 3.5, we use the definition (3.53) extensively in forming our integrations.

The projection map $\pi: \mathfrak{X} \rightarrow|\mathfrak{X}|$ fibering a smooth supermanifold over its reduced space always exists, albeit non-canonically so. This is in contrast to the complex case where holomorphic projections $\pi: \mathfrak{Y} \rightarrow|\mathfrak{Y}|$ need not exist. The formula (3.53) is suitable only in the case where $\mathfrak{Y}$ is such that $\pi$ exists as a holomorphic map. As mentioned earlier, when $\mathfrak{Y}=\overline{\mathfrak{M}}_{g}$ is the supermoduli space of genus $g$ curves, Donagi and Witten in [19] illustrated precisely this: that a holomorphic projection $\pi: \overline{\mathfrak{M}}_{g} \rightarrow\left|\overline{\mathfrak{M}}_{g}\right|$ does not exist when $g \geq 5$.

Another type of object that can be integrated over supermanifolds are integral forms. The integration of integral forms on supermanfolds was discussed in [29, 30, 31]. A particularly appealing feature of the codimension-one integral forms lies in their relation to a generalised Stokes' Theorem. 
Suppose $\mathfrak{X}$ is a supermanifold with boundary $\partial \mathfrak{X}$ and de Rham differential d. Stokes' Theorem asserts that any codimension-one, compactly supported integral form $\nu$ satisfies

$$
\int_{\mathfrak{X}} \mathrm{d} \nu=\int_{\partial \mathfrak{X}} \nu
$$

Specialising to supermoduli space then, for any codimension-one integral form $\nu$ on $\overline{\mathfrak{M}}_{g, n, n^{\prime}}$, we have by (3.54) that $\int_{\overline{\mathfrak{M}}_{g_{1}+g_{2}, n_{1}+n_{1}^{\prime}, n_{2}+n_{2}^{\prime}}} \mathrm{d} \nu=\int_{\partial \overline{\mathfrak{M}}_{g_{1}+g_{2}, n_{1}+n_{1}^{\prime}, n_{2}+n_{2}^{\prime}}} \nu$. By (2.3), the images of $\alpha$ and $\beta$ form components of the boundary $\partial \overline{\mathfrak{M}}_{g, n, n^{\prime}}$. Therefore,

$$
\int_{\overline{\mathfrak{M}}_{g, n, n^{\prime}}} \mathrm{d} \nu=\int_{\partial \overline{\mathfrak{M}}_{g, n, n^{\prime}}} \nu=\int_{\operatorname{im} \alpha} \nu+\int_{\operatorname{im~} \beta} \nu .
$$

In this way we see how codimension-one, integral forms will receive contributions from boundary divisors. Integral forms can also be useful in describing other observables on the boundary of the supermoduli space, such as anomalies.

\subsection{Boundary contribution to three loop vacuum amplitude}

As an illustration of the formalisms in the previous sections, we analyse the three loop vacuum amplitudes in detail in this section. We use the factorisation of super Mumford forms $\Psi_{3}$ near the boundary of the supermoduli space to analyse the contribution to the vacuum amplitude at genus three, from the boundary of the supermoduli space, and consider the cases of NS and Ramond nodes at the degeneration. The supermoduli space of the super Riemann surfaces is denoted by $\overline{\mathfrak{M}}_{g}$, while the spin moduli space of the Riemann surfaces is denoted by $\overline{\mathcal{S M}}_{g}$, and the moduli space of Riemann surfaces is denoted by $\overline{\mathcal{M}}_{g}$.

In this section, $g=g_{1}+g_{2}=3$. Hence the clutching maps describing the degenerations are:

$$
\begin{aligned}
& \overline{\mathfrak{M}}_{2,1} \times \overline{\mathfrak{M}}_{1,1} \stackrel{\alpha_{2,1}}{\longrightarrow} \overline{\mathfrak{M}}_{3,0} . \\
& \overline{\mathfrak{M}}_{3,1} \times \overline{\mathfrak{M}}_{0,1} \stackrel{\alpha_{3,0}}{\longrightarrow} \overline{\mathfrak{M}}_{3,0} . \\
& \overline{\mathfrak{M}}_{2,2} \stackrel{\beta_{2}}{\longrightarrow} \overline{\mathfrak{M}}_{3,0} .
\end{aligned}
$$

Now recall that in genus $g=2$, D'Hoker and Phong constructed a holomorphic projection $\pi_{2,+}: \mathfrak{M}_{2,+} \rightarrow \mathcal{S M}_{2,+}$. This projection can be extended to a meromorphic mapping $\pi_{2,1}: \mathfrak{M}_{2,1} \rightarrow \mathcal{S M}_{2,1}$ which will be meromorphic on the compactification. To retain holomorphy however we can, as in (3.24), specialise the morphism $\bar{q}_{2,1 ;+}$ : $\overline{\mathfrak{M}}_{2,1 ;+} \stackrel{p}{\longrightarrow} \overline{\mathfrak{M}}_{2 ;+} \stackrel{\bar{\pi}_{2 ;+}}{\longrightarrow} \overline{\mathcal{S M}}_{2 ;+}$ to the partial compactification formed by allowing $(+,+)$ 
NS nodes. With the clutching maps above, we obtain a diagram

$$
\begin{aligned}
\overline{\mathfrak{M}}_{2,1 ;+} & \times \overline{\mathfrak{M}}_{1,1} \stackrel{\alpha}{\longrightarrow} \overline{\mathfrak{M}}_{3} \\
& \downarrow \bar{p}_{2} \\
\overline{\mathfrak{M}}_{2 ;+} & \times \overline{\mathfrak{M}}_{1,1} \\
& \downarrow \bar{\pi}_{2 ;+} \times \bar{\pi}_{1,1} \\
\overline{\mathcal{S M}}_{2 ;+} & \times \overline{\mathcal{S M}}_{1,1}
\end{aligned}
$$

The D'Hoker-Phong projection $\pi_{2,+}: \mathfrak{M}_{2,+} \rightarrow \mathcal{S M}_{2,+}$ is defined by sending a genus $g=2$ super Riemann surface with prescribed period matrix to a genus $g=2$ Riemann surface with the same period matrix. This mapping generalises to define mappings $\bar{q}_{2,1 ;+} \times \bar{\pi}_{1,1}$ in the diagram above and a projection $\bar{\pi}_{3}: \overline{\mathfrak{M}}_{3} \rightarrow \overline{\mathcal{S M}}_{3}$. However, we do not consider $\bar{\pi}_{3}$ here since it is meromorphic. As described in Section 3.3, near the boundary of supermoduli space the super Mumford form admits a factorisation as follows:

$$
\begin{aligned}
\left.\Psi_{3}\right|_{\operatorname{im} \alpha_{2,1}} & \sim \Psi_{2,1} F(\varepsilon) \Psi_{1,1} . \\
\left.\Psi_{3}\right|_{\text {im } \alpha_{3,0}} & \sim \Psi_{3,1} F(\varepsilon) \Psi_{0,1} . \\
\left.\Psi_{3}\right|_{\text {im } \beta_{2}} & \sim \Psi_{2,2} G(\varepsilon) .
\end{aligned}
$$

Here, the $\varepsilon$ is the degeneration parameter near the boundary, and $\Psi_{2,1}=p^{*}\left(\Psi_{2}\right)$ for $p$ the forgetful morphism. Note that while $\bar{\pi}_{3 *}\left(\Psi_{3}\right)$ will be singular, $\bar{\pi}_{1,1 *}\left(\Psi_{1,1}\right)$ will not be singular and $\bar{\pi}_{2 ;+*}\left(\Psi_{2}\right)$ will be non-singular along the $(+,+)$ separating NS divisor. As a result, factors such as $\bar{q}_{2,1 ;+*}\left(\Psi_{2,1}\right)$ can be calculated via the D'Hoker-Phong method.

In terms of the gluing parameters $\varepsilon$ and local coordinates near the punctures $a \mid \alpha$ and $b \mid \beta$, we have more explicitly:

$$
\begin{aligned}
\left.\Psi_{3}\right|_{\text {im } \alpha_{2,1}} & \sim \Psi_{2}[\mathrm{~d} a \mid \mathrm{d} \alpha] \frac{\mathrm{d} \varepsilon}{\varepsilon^{2}}[\mathrm{~d} b \mid \mathrm{d} \beta] \Psi_{1} . \\
\left.\Psi_{3}\right|_{\operatorname{im} \alpha_{3,0}} & \sim \Psi_{3}[\mathrm{~d} a \mid \mathrm{d} \alpha] \frac{\mathrm{d} \varepsilon}{\varepsilon^{2}}[\mathrm{~d} b \mid \mathrm{d} \beta] \Psi_{0} . \\
\left.\Psi_{3}\right|_{\operatorname{im} \beta_{2}} & \sim \Psi_{2}[\mathrm{~d} a \mid \mathrm{d} \alpha] \frac{\mathrm{d} \varepsilon}{\varepsilon^{2}}[\mathrm{~d} b \mid \mathrm{d} \beta] .
\end{aligned}
$$

Changing variable from $\varepsilon$ to $q$ as (3.38) and integrating over $\alpha$ and $\beta$ with fixed $q$ then gives:

$$
\begin{aligned}
\left.\Psi_{3}\right|_{\text {im } \alpha_{2,1}} & \sim \Psi_{2,1} \frac{\mathrm{d} q}{q^{2}} \Psi_{1,1} . \\
\left.\Psi_{3}\right|_{\text {im } \alpha_{3,0}} & \sim \Psi_{3,1} \frac{\mathrm{d} q}{q^{2}} \Psi_{0,1} . \\
\left.\Psi_{3}\right|_{\text {im } \beta_{2}} & \sim \Psi_{2,2} \frac{\mathrm{d} q}{q^{2}} .
\end{aligned}
$$


Note, we have used forgetful morphisms in defining the above forms, e.g., $\Psi_{2,1}=p^{*} \Psi_{2}$.

For non-separating degeneration of Ramond type,

$$
\left.\Psi_{3}\right|_{\text {im } \beta_{2}} \sim \sum_{\alpha_{1}, \alpha_{2}} \Psi_{2,2^{\prime}}\left(\Xi_{\alpha_{1}}, \Xi_{\alpha_{2}}\right) e^{\zeta G_{0}}[\mathrm{~d} \zeta] \frac{\mathrm{d} q_{R}}{q_{R}}
$$

where $\Psi_{2,2^{\prime}}\left(\Xi_{\alpha_{1}}, \Xi_{\alpha_{2}}\right)$ is the super Mumford form for a genus 2 super Riemann surface with $n^{\prime}=2$ Ramond punctures and with superconformal primary operators $\Xi_{\alpha_{1}}, \Xi_{\alpha_{2}}$ inserted at these punctures. The terms $q_{R}$ and $\zeta$ are bosonic and fermionic gluing parameters.

The bosonic gluing parameters $\varepsilon$ or $q_{R}$ can be viewed as the bosonic coordinate of the fiber of the normal bundle of the boundary component, in the separating or nonseparating cases respectively, c.f., (2.11).

In the case of an odd spin structure, the superstring vacuum amplitude is zero, since one needs the insertion of operators to absorb the ten fermionic zero-modes of the RNS fermions and for the vacuum amplitude there is no such operator insertions on the genus three surface. Hence for the three loop vacuum amplitude, it suffices to consider genus three surfaces with even spin structures.

The contribution to the superstring amplitude from the boundary of the supermoduli space is the integration as in (2.19),

$$
\mathcal{A}:=\int_{\partial \overline{\mathfrak{M}}_{g}} \int_{\mathfrak{N}} F_{g}
$$

We shall denote by $N$ the reduced space of the fiber $\mathfrak{N}$ of the normal bundle to the boundary divisor.

For general $g_{1}$ and $g_{2}$ with $g_{1}+g_{2}=g=3$ then, we can use the factorisation of the super Mumford form from (3.66)-(3.68) to get,

$$
\mathcal{A}=\int_{\overline{\mathfrak{M}}_{g_{1}, 1} \times \overline{\mathfrak{M}}_{g_{2}, 1}} \int_{\mathfrak{N}} \Psi_{g_{1}, 1} \frac{\mathrm{d} q}{q^{2}} \Psi_{g_{2}, 1} .
$$

It is known for $g \leq 2$, massless tadpole graphs, i.e. one-point functions, all vanish in type II superstring theory with unbroken spacetime supersymmetry [20, 17, 7]. That is, e.g.,

$$
\int_{\overline{\mathfrak{M}}_{g_{1}, 1}} \Psi_{g_{1}, 1}=0
$$

Therefore, since the factor in (3.71) contains a tadpole graph, the amplitude, i.e. the full integral (3.71) will vanish. Now in the expression (3.71), recall that $\Psi_{g_{i}, 1}=p^{*}\left(\Psi_{g_{i}}\right)$, where $p: \overline{\mathfrak{M}}_{g_{i, 1}} \rightarrow \overline{\mathfrak{M}}_{g_{i}}$ is the forgetful morphism. Pushing the measure forward under $p$ then gives $\int_{\overline{\mathfrak{M}}_{g_{i}, 1}} p^{*}\left(\Psi_{g_{i}}\right)=\int_{\overline{\mathfrak{M}}_{g_{i}}} \Psi_{g_{i}}$, c.f., (3.16). The amplitude in (3.71) for separating 
degenerations therefore reduces to,

$$
\mathcal{A}=\int_{\overline{\mathfrak{M}}_{g_{1}} \times \overline{\mathfrak{M}}_{g_{2}}} \int_{\mathfrak{N}} \Psi_{g_{1}} \frac{\mathrm{d} q}{q^{2}} \Psi_{g_{2}}
$$

For non-separating degenerations of the NS type we have,

$$
\mathcal{A}=\int_{\overline{\mathfrak{M}}_{g-1}} \int_{\mathfrak{N}} \Psi_{g-1} \frac{\mathrm{d} q}{q^{2}},
$$

where we have used $\int_{\overline{\mathfrak{M}}_{g-1,2}} \Psi_{g-1,2}=\int_{\overline{\mathfrak{M}}_{g-1,2}} p^{*} p^{*}\left(\Psi_{g-1}\right)$.

For non-separating degenerations of Ramond type,

$$
\mathcal{A}=\int_{\overline{\mathfrak{M}}_{g-1,2^{\prime}}} \int_{\mathfrak{N}} \int_{\mathbb{C}^{0 \mid 1}} \sum_{\alpha_{1}, \alpha_{2}} \Psi_{g-1,2^{\prime}}\left(\Xi_{\alpha_{1}}, \Xi_{\alpha_{2}}\right) e^{\zeta G_{0}}[d \zeta] \frac{\mathrm{d} q_{R}}{q_{R}} .
$$

We have the factorisation by the factor $\int_{\mathfrak{N}} \int_{\mathbb{C}^{0 \mid 1}} e^{\zeta G_{0}}[\mathrm{~d} \zeta] \frac{\mathrm{d} q_{R}}{q_{R}}$. This factor can be holomorphically projected to its reduced space. The integration of $q_{R}$ will be as follows. With an infrared regulator $\epsilon, \int_{N} \frac{\mathrm{d}^{2} q_{R}}{q_{R} \bar{q}_{R}} \sim \int_{\epsilon \leq\left|q_{R}\right|} \frac{\mathrm{d}^{2} q_{R}}{q_{R} \bar{q}_{R}} \sim \ln \epsilon$ could have had a $\ln \epsilon$ infrared divergence, however, the other prefactor would be vanishing due to summations of superconformal operators in the presence of unbroken spacetime supersymmetry.

When $g_{1}, g-1$, or $g_{2} \leq 2$, the projections $\bar{\pi}_{2 ;+}, \bar{\pi}_{1,1}$ and $\bar{\pi}_{0,1}$ to the reduced spaces (which we denote generally by $\pi$ ) are holomorphic, as we discussed in Section 3.2. Note that, for instance, $\bar{\pi}_{1,1}$ being holomorphic implies that $\bar{\pi}_{1,0}$ is also holomorphic. Hence we can use an alternative method by holomorphic projection of one of the factors involving the lower genus supermoduli space and use the integration formula (3.53) , as we describe as follows. Here $N$ is the reduced space of the fiber $\mathfrak{N}$ of the normal bundle of the boundary divisor. Because one of the components of the Riemann surface is a tadpole graph, and the factor of the tadpole graph vanishes, we have e.g.,

$$
\int_{\overline{\mathcal{M}}_{g_{1}}} \pi_{*} \Psi_{g_{1}} \overline{\pi_{*} \Psi_{g_{1}}} \int_{N} \frac{\mathrm{d}^{2} q}{q^{2} \bar{q}^{2}}=0 .
$$

With an infrared regulator $\epsilon, \int_{N} \frac{\mathrm{d}^{2} q}{q^{2} \bar{q}^{2}} \sim \int_{\epsilon \leq|q|} \frac{\mathrm{d}^{2} q}{q^{2} \bar{q}^{2}} \sim \frac{1}{\epsilon^{2}}$ could have had a $\frac{1}{\epsilon^{2}}$ infrared divergence, but due to the vanishing of the prefactor which is a vanishing tadpole graph as in Eq. (3.72), the full integral (3.76) will be vanishing. For the clutching of the second type, with NS degeneration,

$$
\mathcal{A}=\int_{\overline{\mathcal{M}}_{g-1}} \int_{N} \pi_{*} \Psi_{g-1} \overline{\pi_{*} \Psi_{g-1}} \frac{\mathrm{d}^{2} q}{q^{2} \bar{q}^{2}}
$$

D'Hoker and Phong have shown that the two-loop two-point function for massless NS sector vanish, implying that $\int_{\overline{\mathcal{M}}_{g-1}} \pi_{*} \Psi_{g-1} \overline{\pi_{*} \Psi_{g-1}}=0$. Hence the full integral (3.77) is vanishing. 
We also mention that in the case $g_{1}=2, g_{2}=1$, we can project both factors. Then by the integration formula (3.53),

$$
\mathcal{A}=\int_{\overline{\mathcal{M}}_{g_{1}} \times \overline{\mathcal{M}}_{g_{2}}} \int_{N} \pi_{*} \Psi_{g_{1}} \overline{\pi_{*} \Psi_{g_{1}}} \frac{\mathrm{d}^{2} q}{q^{2} \bar{q}^{2}} \pi_{*} \Psi_{g_{2}} \overline{\pi_{*} \Psi_{g_{2}}},
$$

which also shows the vanishing.

There are two methods of computations above. We could first factorise and then

project on the reduced space of one of the factors. For some graphs, we could also first factorise and then project both factors. The two methods are different ways of going along the arrows in the diagram (3.59) as we illustrated.

Our analysis shows that the boundary contribution to the three-loop vacuum amplitude, from the boundary of the supermoduli space will vanish in closed oriented type II superstring theory with unbroken spacetime supersymmetry. Furthermore, we know that the vacuum amplitudes at genus zero, one and two are also vanishing [20, 17, 7], in the closed oriented type II superstring theory, with unbroken spacetime supersymmetry. Here, what we have analysed is the boundary contribution to the superstring amplitude, not the bulk contribution.

There is an analysis of the superstring amplitude at three loop order from the bulk of the bosonic moduli space using modular forms [32, 33, 34, 35, 36]. These results are compatible with our analysis. However the approach there is not derived from supermoduli space or from manifest supersymmetry. It is therefore not obvious how to directly relate their bosonic ansatz with the approach here through supermoduli space.

By similar calculations as presented in this section, we can deduce the following remark.

Remark 3.1. In closed oriented type II superstring theory in spacetime backgrounds with unbroken supersymmetry, the contribution to massless tadpole graphs, i.e. onepoint functions, from the boundary of the supermoduli space, is vanishing at three-loop order.

The idea behind the above remark is, since the Ramond puncture number are always even, one only need to add one additional NS puncture on one component of the super Riemann surface with lower genus. Again one uses the factorisations. One could also use the forgetful morphism associated to that NS puncture, which would be equivalent to the formalism of integrated NS vertex operator.

\section{Discussion}

One of the main goals in this paper is to obtain an understanding of scattering amplitudes in perturbative superstring theory. We focussed in particular on contributions to the superstring amplitude from the boundary of supermoduli space, which in turn can 
be described by clutching morphisms on supermoduli spaces of generally lower genera. The physical interpretation of these clutching morphisms is that they are related to taking an infrared or large distance limit of superstring amplitudes. Fundamentally, the superstring amplitudes can be calculated by integrating the superstring measure, which itself defines a measure on supermoduli space. Following earlier work in [6, 8], we discussed how this measure could be constructed from the generalisation to supermoduli space of the classical Mumford isomorphisms on the moduli space of Riemann surfaces.

The superstring measure, or super Mumford form, is a measure defined on supermoduli space. It is holomorphic on the bulk but, in the compactification, acquires poles near some components of the boundary. We can understand this pole behaviour by looking at how the super Mumford form would factorise into forms over lower genera supermoduli spaces along specified boundary components.

In genus two, and so at two loop order, D'Hoker and Phong calculated the superstring amplitude by integrating the superstring measure over the genus two supermoduli space. This integration involved a projection of supermoduli space onto its reduced, bosonic space, where more classical integration methods could then be applied. In genus three, i.e., at three loop order, it was not clear how to apply D'Hoker and Phong's method of calculation since it is not known whether there exists a holomorphic projection of the genus three supermoduli space onto its reduced, bosonic space. We observe however that since the boundary in genus three parametrises super Riemann surfaces of lower genera, some of these components may at least admit a holomorphic map to a bosonic space. Considering only those boundary components which admit such a map led to the notion of a partial compactification. Following observations by Witten in [8] we note that the genus two boundary component parametrising $(++)$ Neveu-Schwarz degenerations of a genus three super Riemann surface admits precisely such a map.

To state our result more clearly, our analysis shows that the boundary contribution to the three loop vacuum amplitude, from the boundary of the supermoduli space, will vanish in closed, oriented, Type II superstring theory with unbroken spacetime supersymmetry. It also implies that upon compactification to four spacetime dimensions preserving the supersymmetry, the boundary contribution to the cosmological constant at three loop level is also zero in Type II superstring with unbroken spacetime supersymmetry.

Furthermore, our observations are compatible with the results on superstring amplitudes at three loop order in [32, 33, 34, 35, 36] which are obtained using ansatz for modular forms on bosonic moduli space. It is an open and interesting problem as to how to understand the bulk, or interior, contribution to the three loop vacuum amplitude from the viewpoint of supermoduli space. Moreover, it would also be desirable to understand the relation between the approach from supermoduli space and from modular forms in the calculation of superstring amplitudes.

In theories with unbroken supersymmetry the vacuum energy is vanishing since contributions from bosons and fermions cancel each other exactly. It would also be good to 
understand the vanishing of boundary contributions from the point of view of possible nonrenormalisation theorems [37, 38] at three loop, as well as from the point of view of, and relations to, other superstring formalisms such as the Green-Schwarz formalism and the pure spinor formalism.

The boundary contributions to the superstring amplitude can also be viewed as correction terms to calculations of superstring amplitudes over the bulk. Correction terms in genus three may then be relevant for heterotic string theory. Calculations in heterotic string theory are more subtle however, as they involve embedded integration cycles in products of moduli spaces $\mathfrak{M}_{g ; L} \times \mathcal{M}_{g ; R}$ parametrising left and right movers [39, 40]. Boundary contributions, or correction terms, may also be relevant in the calculation of amplitudes for open strings in type II string theory, where there is only one factor of the chiral measure. In the $\alpha^{\prime} \rightarrow 0$ limit one can recover scattering amplitudes for ordinary non-abelian gauge field theories.

More generally and as mentioned earlier, the boundary of the supermoduli space is useful in computing the infrared or large distance limit of the superstring amplitudes. In this limit there appear infinitely thin cylinders forming an internal component of the worldsheet super Riemann surface. Contributions to amplitudes from the boundary of supermoduli space have also been previously considered by e.g., [39, 40, 18, 7, 41]

Fayet-Iliopoulos terms can be generated at one-loop in superstring theory on heterotic backgrounds with anomalous $U(1)$ symmetry [42, 43, 44]. As a consequence, it generates a nonzero mass term at one-loop for tree-level massless scalars charged under the anomalous $U(1)$. It also induces a nonzero dilaton tadpole and nonzero vacuum energy [40, 39] at two-loop. There is tree-level spacetime supersymmetry but it is breaking at one-loop. The aspect of nonzero tadpole amplitudes and associated spacetime supersymmetry breaking by string loop effects is a fundamental idea in superstring theory. A similar degeneration of super Riemann surfaces where there are infinitely thin cylinders can also occur, e.g., as in [44], and so are related to the boundary of supermoduli spaces. As such, these models are particularly relevant for approaches involving supermoduli space and its boundary.

In this paper we have constrained our analysis to Type II superstring theory. The analysis for heterotic $S O(32)$ string theory is more involved [39, 40]. In that case, for certain orbifold backgrounds with tree-level supersymmetry, spacetime supersymmetry is broken at one-loop and there is non-zero two-loop vacuum amplitude [40, 39]. This is mainly due to the breaking of supersymmetry by string loop effects. One main difference is that in the type II theory with unbroken spacetime supersymmetry, we have zero tadpole amplitudes for stable massless particles.

Factorisations of two loop superstring amplitudes also occur in [45] in the type II case, which is useful for checking S-duality of type II superstring theory. The techniques of [45] are also useful in computing the normalisation factor for the vacuum energy. The S-duality covariance and factorisation constraints would be a good consistency check for computing the amplitudes and the effective action in Type II superstring and 
supergravity theory.

\section{Acknowledgments}

The work was supported in part by Yau Mathematical Sciences Center and Tsinghua University, and by grant TH-533310008 of Tsinghua University (to H.L.).

\section{A Compactification of moduli space and spin mod- uli space}

In this appendix, in order to be self-contained and for the convenience of the reader, we briefly overview the compactification of the moduli space of ordinary Riemann surfaces and the compactification of the spin moduli space of spin Rieman surfaces. Note that we use the term Riemann surface interchangeably with (complex) curve.

The moduli space $\mathcal{M}_{g}$ parametrises families of smooth curves $\mathcal{X}$ over a base $B$. The non-compactness of $\mathcal{M}_{g}$ follows from the property that such families a non-complete base $B$ cannot generally be extended to smooth families of curves over the completion $\widehat{B}$, even up to finitely many base-changes. Remarkably however, if one allows families of curves to have at-worst nodal singularities, then any family of smooth curves $\mathcal{X} \rightarrow B$ can be extended to a family of stable curves $\widehat{\mathcal{X}} \rightarrow \widehat{B}$ with at-worst nodal singularities. A stable curve $C$ has a nodal singularity at a point $p \in C$ if, in any local, affine coordinate system $(x, y)$ at $p$ that $x y=0$ at $p$. The boundary divisor of the compactification $\partial \overline{\mathcal{M}}_{g} \subset$ $\overline{\mathcal{M}}_{g}$ consists of degenerations of two distinct kinds: separating and non-separating. If $D_{\text {sep. }} \subset \partial \overline{\mathcal{M}}_{g}$ parametrises separating degenerations, then a generic component of $D_{\text {sep }}$. is isomorphic to the product $\overline{\mathcal{M}}_{g_{1}, 1} \times \overline{\mathcal{M}}_{g_{2}, 1}$ where $g_{1}+g_{2}=g$. If $D_{n s e p} \subset \partial \overline{\mathcal{M}}_{g}$ parametrises non-separating degenerations, then $D_{\text {nsep. }} \cong \overline{\mathcal{M}}_{g-1,2}$. More generally, with $\overline{\mathcal{M}}_{g, n}$ the Deligne-Mumford compactification of $n$-pointed, stable, genus $g$ curves, its boundary $\partial \overline{\mathcal{M}}_{g, n}$ will also parametrise separating and non-separating degenerations.

With $D_{\text {sep. }} \subset \partial \overline{\mathcal{M}}_{g, n}$ parametrising the separating degenerations, a generic component is isomorphic to the product $\overline{\mathcal{M}}_{g_{1}, n_{1}+1} \times \overline{\mathcal{M}}_{g_{2}, n_{2}+1}$ where $n_{1}+n_{2}=n$. With $D_{\text {nsep }} \subset \partial \overline{\mathcal{M}}_{g, n}$ parametrising the non-separating degenerations, $D_{n s e p .} \cong \overline{\mathcal{M}}_{g-1, n+2}$. The morphisms on boundary components induced by the inclusion $\partial \overline{\mathcal{M}}_{g, n} \subset \overline{\mathcal{M}}_{g, n}$,

$$
\overline{\mathcal{M}}_{g_{1}, n_{1}+1} \times \overline{\mathcal{M}}_{g_{2}, n_{2}+1} \stackrel{\alpha}{\longrightarrow} \overline{\mathcal{M}}_{g, n} \text { and } \overline{\mathcal{M}}_{g-1, n+2} \stackrel{\beta}{\longrightarrow} \overline{\mathcal{M}}_{g, n}
$$

are referred to as $\alpha$ - and $\beta$-clutchings respectively.

A spin curve is a smooth curve $C$ equipped with a choice of spin structure $L_{C}$, which is a line bundle over $C$ satisfying $L_{C}^{\otimes 2} \cong \omega_{C}$ for $\omega_{C}$ the canonical bundle. The notion of stable, pointed curves $C$ can be generalised to stable pointed spin curves $\left(C, L_{C}\right)$. 
Here, $L_{C}$ restricts to a spin structure $L_{C}$ sm. on the smooth locus of the stable curve $C^{s m} \subset C$, and vanishes along the nodes of $C$. Blowing up along the nodes $\left\{p_{i}\right\} \in C$ results in a smooth curve $\pi: \widetilde{C} \rightarrow C$. Off the exceptional divisor, $\widetilde{C} \backslash \pi^{-1}\left(\left\{p_{i}\right\}\right)$ is isomorphic to the smooth locus $C^{s m}$. As such, the spin structure $L_{C^{s m}}$. on $C^{s m}$. pulls back to $\widetilde{C} \backslash \pi^{-1}\left(\left\{p_{i}\right\}\right)$. The exceptional divisor $E=\pi^{-1}\left(\left\{p_{i}\right\}\right) \subset \widetilde{C}$ is isomorphic to a product of $\mathbb{P}_{\mathbb{C}}^{1}$ 's and so the spin structure $L_{C^{s m}}$. can be continued along $E$ by gluing in the standard spin structure $\mathcal{O}_{\mathbb{P}_{\mathbb{C}}^{1}}(-1)$ on $\mathbb{P}_{\mathbb{C}}^{1}$. Cornalba's compactification $\overline{\mathcal{S M}}_{g, n}$ is holomorphically fibered over $\overline{\mathcal{M}}_{g, n}$ with boundary components comprising clutchings, in analogy with (A.1). In contrast to $\overline{\mathcal{M}}_{g}$ however, the spin structures $L_{C}$ are themselves endowed with parity: $L_{C}$ is even or odd if $h^{0}\left(C, L_{C}\right) \equiv 0$ or $h^{0}\left(C, L_{C}\right) \equiv 1 \bmod 2$, respectively. The parity of spin structures are invariant under continuous deformation. This leads to a decomposition of the spin moduli space, $\mathcal{S M}_{g}=\mathcal{S} \mathcal{M}_{g}^{+} \cup \mathcal{S} \mathcal{M}_{g}^{-}$. In the compactification of $\overline{\mathcal{S M}}_{g}$, one needs therefore to account for the parity of the spin structure in the separating degenerations at a node. The analogue of the $\alpha$-clutchings in (A.1) making up the boundary components $\partial \overline{\mathcal{S M}}_{g, n} \subset \overline{\mathcal{S M}}_{g, n}^{+}$are therefore:

$$
\overline{\mathcal{S M}}_{g_{1}, n_{1}+1}^{+} \times \overline{\mathcal{S M}}_{g_{1}, n_{2}+1}^{+} \stackrel{\alpha^{++}}{\longrightarrow} \overline{\mathcal{S M}}_{g, n}^{+} \text {and } \overline{\mathcal{S M}}_{g_{1}, n_{1}+1}^{-} \times \overline{\mathcal{S M}}_{g_{1}, n_{2}+1}^{-} \stackrel{\alpha^{--}}{\longrightarrow} \overline{\mathcal{S M}}_{g, n}^{+}
$$

where $g_{1}+g_{2}=g$ and $n_{1}+n_{2}=n$. Similarly, the $\beta$-clutching is given by:

$$
\overline{\mathcal{S M}}_{g-1,2}^{+} \stackrel{\beta^{+}}{\longrightarrow} \overline{\mathcal{S M}}_{g, n}^{+}
$$

Since clutchings are natural in families, both the $\alpha$ - and $\beta$-clutchings on the spin moduli

space and the moduli space respectively are compatible with the holomorphic fibration $\overline{\mathcal{S M}}_{g} \rightarrow \overline{\mathcal{M}}_{g}$.

\section{References}

[1] F. A. Berezin, Introduction to Superanalysis, D. Reidel Publ., 1987.

[2] C. Bartocci, U. Bruzzo and D. Hernandez-Ruiperez, The Geometry of Supermanifolds, Kluwer Academic Publ., 1991.

[3] T. Voronov, Geometric Integration Theory on Supermanifolds, Sov. Sci. Rev. C. Math. Phys., vol. 9, Harwood Academic Publ., 1991.

[4] A. A. Belavin and V. G. Knizhnik, "Algebraic Geometry and the Geometry of Quantum Strings," Phys. Lett. B 168 (1986), 201-206.

[5] A. A. Beilinson and Y. I. Manin, "The Mumford Form and the Polyakov Measure in String Theory," Commun. Math. Phys. 107 (1986), 359-376. 
[6] A. A. Rosly, A. S. Schwarz and A. A. Voronov, "Superconformal Geometry and String Theory," Commun. Math. Phys. 120 (1989), 437.

[7] E. Witten, "Superstring Perturbation Theory Revisited," [arXiv:1209.5461 [hepth]].

[8] E. Witten, "Notes On Holomorphic String And Superstring Theory Measures Of Low Genus," Pure Appl. Math. Q. 15 (2019), no. 1, 3-56 [arXiv:1306.3621 [hep-th]].

[9] D. Diroff, "On the super Mumford form in the presence of Ramond and NeveuSchwarz punctures," J. Geom. Phys. 144 (2019), 273-293 [arXiv:1802.07865 [math$\mathrm{ph}]]$.

[10] E. D'Hoker and D. H. Phong, "The Geometry of String Perturbation Theory," Rev. Mod. Phys. 60 (1988), 917.

[11] S. B. Giddings, "Conformal Techniques in String Theory and String Field Theory," Phys. Rept. 170 (1988), 167.

[12] D. Friedan, S. H. Shenker and E. J. Martinec, "Covariant Quantization of Superstrings," Phys. Lett. B 160 (1985), 55-61.

[13] G. W. Moore, P. C. Nelson and J. Polchinski, "Strings and Supermoduli," Phys. Lett. B 169 (1986), 47.

[14] E. P. Verlinde and H. L. Verlinde, "Multiloop Calculations in Covariant Superstring Theory," Phys. Lett. B 192 (1987), 95-102.

[15] L. Alvarez-Gaume, C. Gomez, P. C. Nelson, G. Sierra and C. Vafa, "Fermionic Strings in the Operator Formalism," Nucl. Phys. B 311 (1988), 333-400.

[16] E. D'Hoker and D. H. Phong, "Conformal Scalar Fields and Chiral Splitting on Super Riemann Surfaces," Commun. Math. Phys. 125 (1989), 469.

[17] E. D'Hoker and D. H. Phong, "Two loop superstrings. I. Main formulas," Phys. Lett. B 529 (2002), 241-255 [arXiv:hep-th/0110247 [hep-th]].

[18] E. Witten, "Notes On Super Riemann Surfaces And Their Moduli," [arXiv:1209.2459 [hep-th]].

[19] R. Donagi and E. Witten, "Supermoduli Space Is Not Projected," Proc. Symp. Pure Math. 90 (2015), 19-71 [arXiv:1304.7798 [hep-th]].

[20] M. B. Green and J. H. Schwarz, "Supersymmetrical String Theories," Phys. Lett. B 109 (1982), 444-448. 
[21] E. Witten, "The Super Period Matrix With Ramond Punctures," J. Geom. Phys. 92 (2015), 210-239 [arXiv:1501.02499 [hep-th]].

[22] R. Donagi and E. Witten, "Super Atiyah classes and obstructions to splitting of supermoduli space," Pure Appl. Math. Q. 9 (2013), no. 4, 739-788 [arXiv:1404.6257 [hep-th]].

[23] F. Knudsen, "The projectivity of the moduli space of stable curves. II.", Math. Scand. 52 (1983), no. 2, 161-199.

[24] E. D'Hoker and D. H. Phong, "The Super Period Matrix with Ramond Punctures in the supergravity formulation," Nucl. Phys. B 899 (2015), 772-809 [arXiv:1501.02675 [hep-th]].

[25] P. Deligne and D. Mumford, "The irreducibility of the space of curves of given genus," Inst. Hautes Etudes Sci. Publ. Math. 36 (1969), 75-109.

[26] M. Cornalba, "Moduli of curves and theta-characteristics," Lectures on Riemann surfaces (Trieste, 1987), 560-589, World Sci. Publ., 1989.

[27] R. Bott and L. W. Tu, Differential Forms in Algebraic Topology, Springer, 1982.

[28] M. Batchelor, "The Structure of Supermanifolds," Trans. Amer. Math. Soc. 253 (1979), 329-338.

[29] J. N. Bernstein, D. A. Leites, "Integral forms and Stokes formula on supermanifolds," Functional Anal. Appl. 11 (1977), no. 1, 45-47.

[30] J. N. Bernstein, D. A. Leites, "How to integrate differential forms on supermanifolds," Functional Anal. Appl. 11 (1977), no. 3, 219-221.

[31] E. Witten, "Notes On Supermanifolds and Integration," [arXiv:1209.2199 [hep-th]].

[32] E. D'Hoker and D. H. Phong, "Asyzygies, modular forms, and the superstring measure. I.," Nucl. Phys. B 710 (2005), 58-82 [arXiv:hep-th/0411159 [hep-th]].

[33] S. L. Cacciatori, F. Dalla Piazza and B. van Geemen, "Modular Forms and Three Loop Superstring Amplitudes," Nucl. Phys. B 800 (2008), 565-590 [arXiv:0801.2543 [hep-th]].

[34] S. Grushevsky, "Superstring scattering amplitudes in higher genus," Commun. Math. Phys. 287 (2009), 749-767 [arXiv:0803.3469 [hep-th]].

[35] M. Matone and R. Volpato, "Getting superstring amplitudes by degenerating Riemann surfaces," Nucl. Phys. B 839 (2010), 21-51 [arXiv:1003.3452 [hep-th]]. 
[36] S. L. Cacciatori, F. Dalla Piazza and B. van Geemen, "Genus four superstring measures," Lett. Math. Phys. 85 (2008), 185-193 [arXiv:0804.0457 [hep-th]].

[37] E. J. Martinec, "Nonrenormalization Theorems and Fermionic String Finiteness," Phys. Lett. B 171 (1986), 189.

[38] M. Dine and N. Seiberg, "Nonrenormalization Theorems in Superstring Theory," Phys. Rev. Lett. 57 (1986), 2625.

[39] E. Witten, "Superstring Perturbation Theory Via Super Riemann Surfaces: An Overview," [arXiv:1304.2832 [hep-th]].

[40] E. D'Hoker and D. H. Phong, "Two-loop vacuum energy for Calabi-Yau orbifold models," Nucl. Phys. B 877 (2013), 343-400 [arXiv:1307.1749 [hep-th]].

[41] A. Sen, "Supersymmetry Restoration in Superstring Perturbation Theory," JHEP 12 (2015), 075 [arXiv:1508.02481 [hep-th]].

[42] M. Dine, N. Seiberg and E. Witten, "Fayet-Iliopoulos Terms in String Theory," Nucl. Phys. B 289 (1987), 589-598.

[43] J. J. Atick, L. J. Dixon and A. Sen, "String Calculation of Fayet-Iliopoulos D Terms in Arbitrary Supersymmetric Compactifications," Nucl. Phys. B 292 (1987), 109149 .

[44] M. Dine, I. Ichinose and N. Seiberg, "F Terms and D Terms in String Theory," Nucl. Phys. B 293 (1987), 253-265.

[45] E. D'Hoker, M. Gutperle and D. H. Phong, "Two-loop superstrings and S-duality," Nucl. Phys. B 722 (2005), 81-118 [arXiv:hep-th/0503180 [hep-th]]. 